\title{
Life Cycle Assessment of A Hydrocarbon-based Electrified Cleaning Agent
}

\author{
Peng Liu ${ }^{1}$, Bo Zhang ${ }^{1 *}$, Changyan Yang ${ }^{1,2^{*}}$, Yu Gong ${ }^{1}$, Yinhang $\mathrm{Qu}^{1}$, Jiayi $\mathrm{Li}^{1}$, Bohan \\ Yang ${ }^{1}$, Yigang Ding ${ }^{1}$ \\ 1: Key Laboratory for Green Chemical Process of Ministry of Education, Hubei Key Laboratory of Novel \\ Chemical Reactor and Green Chemical Technology, School of Chemical Engineering and Pharmacy, \\ Wuhan Institute of Technology, Hubei, China \\ 2: Hubei Key Laboratory for Processing and Application of Catalytic Materials, Huanggang Normal \\ University, Hubei, China
}

Received April 15, 2021; Accepted May 1, 2021; Published May 3, 2021

The electrified cleaning agent requires a moderate volatilization rate, low ozone-depleting substances value, non-flammable, non-explosive and other characteristics. This study performed a whole life cycle assessment on a hydrocarbon-based electrified cleaning agent. The life cycle model is cradle-to-grave, and the background data sets include power grid, transportation, high-density polyethylene, chemicals, etc. The analysis shows that the global warming potential (GWP) of the life cycle of $1 \mathrm{~kg}$ of electrified cleaning agent is $2.08 \mathrm{~kg} \mathrm{CO}_{2}$ eq, acidification potential (AP) is $9.49 \mathrm{E}-03 \mathrm{~kg} \mathrm{SO}{ }_{2}$ eq, eutrophication potential (EP) is $1.18 \mathrm{E}-03 \mathrm{~kg} \mathrm{PO}_{4}{ }^{3-}$ eq, respirable inorganic matter $(\mathrm{RI})$ is $2.13 \mathrm{E}-03 \mathrm{~kg} P M 2.5 \mathrm{eq}$, ozone depletion potential (ODP) is 4.91E-05 kg CFC-11 eq, photochemical ozone formation potential (POFP) is $2.89 \mathrm{E}-02 \mathrm{~kg} \mathrm{NMVOC}$ eq, ionizing radiation-human health potential (IRP) is $3.16 \mathrm{E}-02 \mathrm{~kg} U 235 \mathrm{eq}$, ecotoxicity $(E T)$ is 2.69E-01 CTUe, human toxicity-carcinogenic (HTcancer) is 4.32E-08 CTUh, and human toxicity-non-carcinogenic (HT-non cancer) is 2.31E-07 CTUh. The uncertainty of the results is between $3.46-9.95 \%$.

The four processes of tetrachloroethylene production, D40 solvent oil production, tetrachloroethylene environmental discharge during product use, and electricity usage during product disposal have substantial effects on each LCA indicator, so they are the focus of process improvement. Changes in power consumption during production and transportation distance of raw materials have little effect on total carbon emissions. Compared with the production process of single-solvent electrified cleaning agent tetrachloroethylene and n-bromopropane, the production of the electrified cleaning agent developed in this study has its own advantages in terms of carbon footprint and other environmental impact indicators. Carbon emissions mainly come from the power consumption of each process, natural gas production and combustion, and other energy materials for heating. It is recommended to use renewable raw materials instead of crude oil to obtain carbon credits based on geographical advantages, and try to use production processes with lower carbon emissions, while the exhaust gas from the traditional production process is strictly absorbed and purified before being discharged.

Keywords: D40 solvent oil; Tetrachloroethylene; Global warming potential (GWP), Acidification potential (AP); Eutrophication potential (EP); Photochemical ozone formation potential (POFP); Ecotoxicity (ET) 


\section{Introduction}

Electrified cleaning refers to cleaning operations performed under normal working conditions with a certain voltage. It is usually required that the electrified cleaning agent must have a moderate volatilization rate (i.e., not to freeze the line), low ozone-depleting substances (ODS) value, non-flammable, non-explosive and other characteristics. The electrified cleaning agent is mainly used for cleaning equipment and devices in use under high-voltage electric fields, such as locomotive substations, highvoltage substations and high-voltage lines. Traditional charged cleaning agents mostly use chlorinated hydrocarbons (e.g., Freon), bromo-hydrocarbons and other halogenated hydrocarbons, which are high specific resistance reagents with high ODS value and strong volatility. They may damage the atmospheric $\mathrm{O}_{3}$ layer, and are easy to cause the line to freeze and increase the load of the line, affecting the normal operation [1].

The eco-friendly cleaning agent for high/low voltage equipment in use developed by this study is compounded with D40 solvent oil and tetrachloroethylene with extremely low ODS value as the base liquid, supplemented by butanol and other additives, which can effectively remove soil, dirt, oil stains and animal feces on the surface of charged devices. The electrified cleaning agent has a resistance value of more than $10 \mathrm{G} \mathrm{ohms,}$ and can be used in an electric field environment of up to 100,000 volts. It is mainly used for live cleaning of high-voltage circuits, substations, electrical equipment, etc. This research conducts a full life cycle assessment of the electrified cleaning agent, including the production, use and waste recycling process of the product.

\section{Definition of Objectives and Scope}

\subsection{Object Definition}

\subsubsection{Product Information}

The research object of this study is: to access an electrified cleaning agent. The specific information is as follows :

Specification number : DL1006-series

Product category: Eco-environmental cleaning agent for high/low voltage live equipment (such as high voltage circuit, substation and electrical equipment). The target users of eco-environmental cleaning agents are the customers with high-voltage circuits, substations, electrical equipment and other equipment, including high-speed railways, motor vehicles, aircrafts, tanks, electrical equipment and instruments of ships.

Shape and form: solution

\subsubsection{Functional Unit and Benchmark Flow}

Functional unit: $1 \mathrm{~kg}$ product

\subsubsection{Data Representation}

The representation of time, geography and technology is as follows:

(1) Time representation: 2020

(2) Geographical representation: China 


\subsection{Scope Definitions}

\subsubsection{System Boundary}

The system boundary of this study is from cradle to grave (from resource exploitation to waste treatment and recycle). The life process includes the production, use, recovery and waste solvent distillation of charged cleaners. Background processes include power grid, transportation, high density polyethylene for packaging, chemicals and sludge landfilling.

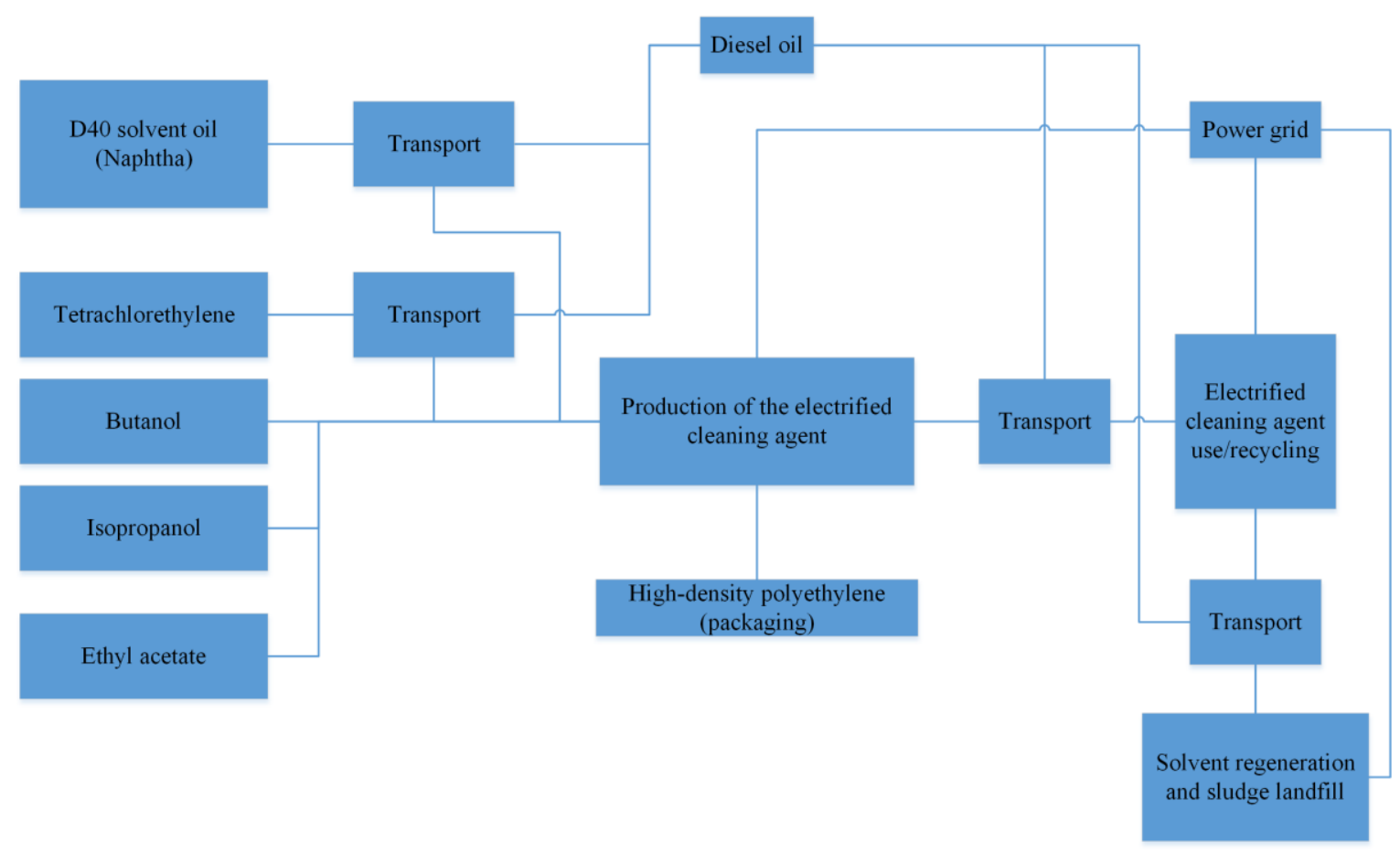

Figure. 1. System boundary of the electrified cleaning agent

\subsubsection{Selection Principle}

The trade-off rules used in this study are based on the weight ratio of raw material to the product weight or the total process input. Specific rules are as follows:

(1) When the weight of ordinary material is less than $1 \%$ of the product weight and the weight of material containing rare or high purity components is less than $0.1 \%$ of the product weight, the upstream production data of the material can be ignored. Total negligible material weight shall not exceeding $5 \%$.

(2) The material can be ignored if the low value waste is used as raw materials, such as fly ash, slag, straw, garbage and other upstream production data. The production equipment, plant, living facilities and other emissions are ignored.

\subsubsection{Environmental Impact Indicators}

Environmental impact indicators are summarized in Table 1. Data quality represents the difference between the target representativeness of LCA research and the actual representativeness of data. The data quality evaluation method in this report adopts the CLCD method. 
Table 1. Environmental impact indicators

\begin{tabular}{|c|c|c|}
\hline $\begin{array}{c}\text { Environmental impact } \\
\text { indicators }\end{array}$ & Indicator unit & Main substances \\
\hline $\begin{array}{c}\text { Global warming potential } \\
\text { (GWP) }\end{array}$ & $\mathrm{kg} \mathrm{CO}_{2}$ eq. & $\mathrm{CO}_{2}, \mathrm{CH}_{4}, \mathrm{~N}_{2} \mathrm{O} \ldots$ \\
\hline Acidify potential (AP) & $\mathrm{kg} \mathrm{SO}_{2}$ eq. & $\mathrm{SO}_{2}, \mathrm{NO}_{\mathrm{x}}, \mathrm{NH}_{3} \ldots$ \\
\hline $\begin{array}{c}\text { Eutrophication potential } \\
\text { (EP) }\end{array}$ & $\mathrm{kg} \mathrm{PO}_{4}{ }^{3-}$ eq. & $\mathrm{NH}_{3}, \mathrm{NH}_{4}-\mathrm{N}, \mathrm{COD} \ldots$ \\
\hline $\begin{array}{l}\text { Inhalable inorganic matter } \\
\text { (RI) }\end{array}$ & kg PM2.5 eq. & $\mathrm{CO}, \mathrm{PM}_{10}, \mathrm{PM}_{2.5} \ldots$ \\
\hline $\begin{array}{l}\text { Ozone depletion potential } \\
\text { (ODP) }\end{array}$ & kg CFC-11 eq. & $\mathrm{CCl}_{4}, \mathrm{C}_{2} \mathrm{H}_{3} \mathrm{Cl}_{3}, \mathrm{CH}_{3} \mathrm{Br} \ldots$ \\
\hline $\begin{array}{c}\text { photochemical ozone } \\
\text { formation potentials(POFP) }\end{array}$ & kg NMVOC eq. & $\mathrm{C}_{2} \mathrm{H}_{6}, \mathrm{C}_{2} \mathrm{H}_{4} \ldots$ \\
\hline $\begin{array}{c}\text { Ionizing Radiation - Human } \\
\text { Health }\end{array}$ & kg U235 eq. & C-14, Cs- $134 \ldots$ \\
\hline Ecotoxicity & CTUe & $\mathrm{HF}, \mathrm{Hg}^{2+}, \mathrm{Be} \ldots$ \\
\hline $\begin{array}{l}\text { Human toxicity - } \\
\text { carcinogenic }\end{array}$ & CTUh & As, $\mathrm{Cr}, \mathrm{Pb} \ldots$ \\
\hline $\begin{array}{l}\text { Human toxicity - } \\
\text { noncarcinogenic }\end{array}$ & CTUh & $\mathrm{Hg}^{2+}, \mathrm{HF}, \mathrm{TI} \ldots$ \\
\hline
\end{tabular}

Note: eq. is the abbreviation of equivalent.

\subsubsection{Software and Database}

In this study, the eFootprint software system was used to establish a life cycle model of charged detergents and calculate the LCA results. The eFootprint software system is an online LCA analysis software developed by IKE Environmental Technology Co., Ltd (http://ike-global.com/). It supports full life cycle process analysis and has builtin China Life Cycle Basic Database (CLCD), European ELCD database and Swiss Ecoinvent database.

The China Life Cycle Basic Database (CLCD) used in the research was developed by IKE, which is an industry average database based on the core model of China's basic industrial system life cycle. The CLCD database includes inventory data sets of major domestic energy, transportation and basic raw materials.

The LCA model of the electrified cleaning agent was established in eFootprint software. The background data sources of this LCA study are shown in Table 2: 
Table 2. Background data source

\begin{tabular}{|c|c|c|c|c|}
\hline List name & $\begin{array}{c}\text { Specificatio } \\
\text { n model }\end{array}$ & Process & Data set name & $\begin{array}{c}\text { Name } \\
\text { database }\end{array}$ \\
\hline Electricity & $\mathrm{AR}$ & $\begin{array}{c}\text { Electrified cleaning } \\
\text { agent } \\
\text { 【Production】 }\end{array}$ & $\begin{array}{l}\text { East China Power } \\
\text { Grid (to users) }\end{array}$ & $\begin{array}{l}\text { CLCD-China- } \\
\text { ECER } 0.8\end{array}$ \\
\hline Electricity & - & $\begin{array}{l}\text { Electrified cleaning } \\
\text { agent【use】 }\end{array}$ & $\begin{array}{l}\text { East China Power } \\
\text { Grid (to users) }\end{array}$ & $\begin{array}{l}\text { CLCD-China- } \\
\text { ECER } 0.8\end{array}$ \\
\hline Electricity & - & $\begin{array}{c}\text { Electrified cleaning } \\
\text { agent } \\
\text { 【Disposal】 }\end{array}$ & $\begin{array}{l}\text { East China Power } \\
\text { Grid (to users) }\end{array}$ & $\begin{array}{l}\text { CLCD-China- } \\
\text { ECER } 0.8\end{array}$ \\
\hline D40 solvent oil & $\mathrm{CP}$ & $\begin{array}{c}\text { Electrified cleaning } \\
\text { agent } \\
\text { 【Production】 }\end{array}$ & Naphtha/Naphtha & $\begin{array}{l}\text { CLCD-China- } \\
\text { ECER } 0.8\end{array}$ \\
\hline Tetrachloroethylene & $\mathrm{AR}$ & $\begin{array}{c}\text { Electrified cleaning } \\
\text { agent } \\
\text { 【Production】 }\end{array}$ & $\begin{array}{l}\text { tetrachloroethylene } \\
\text { production }\end{array}$ & $\begin{array}{c}\text { Ecoinvent } \\
3.1^{[2]}\end{array}$ \\
\hline Isopropanol & $\mathrm{AR}$ & $\begin{array}{c}\text { Electrified cleaning } \\
\text { agent } \\
\text { 【Production】 }\end{array}$ & $\begin{array}{l}\text { Isopropanol } \\
\text { (domestic) }\end{array}$ & $\begin{array}{l}\text { CLCD-China- } \\
\text { ECER } 0.8\end{array}$ \\
\hline Butanol & $\mathrm{AR}$ & $\begin{array}{c}\text { Electrified cleaning } \\
\text { agent } \\
\text { 【Production】 }\end{array}$ & 1-Butanol & Ecoinvent 3.1 \\
\hline Ethyl acetate & $\mathrm{AR}$ & $\begin{array}{c}\text { Electrified cleaning } \\
\text { agent } \\
\text { 【Production】 }\end{array}$ & Ethyl acetate & Ecoinvent 3.1 \\
\hline $\begin{array}{l}\text { High-density } \\
\text { polyethylene }\end{array}$ & - & $\begin{array}{c}\text { Electrified cleaning } \\
\text { agent } \\
\text { 【Production】 }\end{array}$ & $\begin{array}{l}\text { High-density } \\
\text { polyethylene }\end{array}$ & $\begin{array}{l}\text { CLCD-China- } \\
\text { ECER } 0.8\end{array}$ \\
\hline Sludge landfill & - & $\begin{array}{c}\text { Electrified cleaning } \\
\text { agent } \\
\text { 【Disposal】 }\end{array}$ & Landfill of textiles & ELCD 3.0 \\
\hline
\end{tabular}

\section{Data Collection}

\subsection{Electrified Cleaning Agent【Production】}

(1) Basic process information

Process name: electrified cleaning agent【production】

Process boundary: from cradle to gate

(2) Data representativeness

Main data sources: chemical process simulation, enterprise surveys. The data does not represent industry average data

Company name: Suqian Dunjia Biotechnology Co., Ltd. (Jiangsu, China)

Origin: China

Base year: 2020

Process equipment: reactors, steam generator, vacuum homogenizer, packaging machine

Main raw material: organic solvent

Main energy consumption: electricity 
Production scale: $2000 \mathrm{t} / \mathrm{y}$

Table 3 LCI Data of the electrified cleaner 【production】 process

\begin{tabular}{|c|c|c|c|c|c|}
\hline Type & List name & Quantity & unit & $\begin{array}{l}\text { Upstream data } \\
\text { source }\end{array}$ & $\begin{array}{l}\text { Use / emission } \\
\text { reasons }\end{array}$ \\
\hline Product & $\begin{array}{c}\text { Electrified } \\
\text { cleaning agent }\end{array}$ & 1 & $\mathrm{~kg}$ & -- & -- \\
\hline Raw materials & D40 solvent oil & 0.64 & $\mathrm{~kg}$ & $\begin{array}{l}\text { CLCD-China- } \\
\text { ECER } 0.8^{[3]}\end{array}$ & \\
\hline Raw materials & Tetrachlorethylene & 0.33 & $\mathrm{~kg}$ & Ecoinvent 3.1 & \\
\hline Raw materials & Butanol & 0.01 & $\mathrm{~kg}$ & Ecoinvent 3.1 & \\
\hline Raw materials & Isopropanol & 0.02 & $\mathrm{~kg}$ & $\begin{array}{l}\text { CLCD-China- } \\
\text { ECER } 0.8^{[4]}\end{array}$ & \\
\hline Raw materials & Ethyl acetate & $3.62 \mathrm{E}-03$ & $\mathrm{~kg}$ & Ecoinvent 3.1 & \\
\hline Raw materials & $\begin{array}{l}\text { High-density } \\
\text { polyethylene }\end{array}$ & 0.02 & $\mathrm{~kg}$ & $\begin{array}{l}\text { CLCD-China- } \\
\text { ECER } 0.8\end{array}$ & \\
\hline Energy & electricity & 0.05 & MJ & $\begin{array}{l}\text { CLCD-China- } \\
\text { ECER } 0.8^{[5]}\end{array}$ & \\
\hline $\begin{array}{l}\text { Environmental } \\
\text { emissions }\end{array}$ & water vapor & $8.40 \mathrm{E}-04$ & $\mathrm{~kg}$ & & $\begin{array}{c}\text { Boiler } \\
\text { blowdown }\end{array}$ \\
\hline $\begin{array}{l}\text { Environmental } \\
\text { emissions }\end{array}$ & $\begin{array}{l}\text { Suspended solids, } \\
\text { unspecified }\end{array}$ & $9.33 \mathrm{E}-05$ & $\mathrm{~kg}$ & & $\begin{array}{l}\text { Discharge (body } \\
\text { of water) }\end{array}$ \\
\hline $\begin{array}{l}\text { Environmental } \\
\text { emissions }\end{array}$ & $\begin{array}{l}\text { biological oxygen } \\
\text { demand }\end{array}$ & 0.0001 & $\mathrm{~kg}$ & & $\begin{array}{l}\text { Discharge (body } \\
\text { of water) }\end{array}$ \\
\hline $\begin{array}{c}\text { Environmental } \\
\text { emissions }\end{array}$ & COD & 0.0001 & $\mathrm{~kg}$ & & $\begin{array}{l}\text { Discharge (body } \\
\text { of water) }\end{array}$ \\
\hline
\end{tabular}

\section{(3) Transportation information}

Table 4. Transportation information

\begin{tabular}{cccccc}
\hline Material name & $\begin{array}{c}\text { Gross } \\
\text { weight }\end{array}$ & $\begin{array}{c}\text { Starting } \\
\text { point }\end{array}$ & End & $\begin{array}{c}\text { Transport } \\
\text { distance }\end{array}$ & $\begin{array}{c}\text { Type of } \\
\text { transport }\end{array}$ \\
\hline D40 solvent oil & $1.1 \mathrm{~kg}$ & Jiangsu & Jiangsu & $200 \mathrm{~km}$ & $\begin{array}{c}\text { Truck } \\
\text { transportation } \\
(30 \mathrm{t}) \text {-diesel } \\
\text { Truck }\end{array}$ \\
Tetrachloroethylene & $1.1 \mathrm{~kg}$ & Jiangsu & Jiangsu & $200 \mathrm{~km}$ & $\begin{array}{c}\text { transportation } \\
\text { (30t)-diesel }\end{array}$ \\
\hline
\end{tabular}

Note: In the benchmark model, only the transportation of D40 solvent oil and tetrachloroethylene, which account for the largest proportion, is considered. The upstream data sources of transportation data are all from the CLCD database.

Technical supplementary description: The production is planned to use the existing equipment of a cleaning agent company in Jiangsu Suqian Dunjia Biotechnology Co., Ltd. The strict material and energy consumption balance calculations for the production process was performed by using ASPEN Plus. Figure 2 shows the flow chart of the simulation. D40 solvent oil and tetrachloroethylene (PCE), which are main raw materials of the charged cleaner, are added to the stirred tank 1 through pumps 1 and 2 (PUMP1 and PUMP2), respectively. The material is heated to $30^{\circ} \mathrm{C}$ by steam and stirred at $30^{\circ} \mathrm{C}$ for 30 minutes. After mixing, the materials are pumped by the pump 3 to the heat 
exchanger to cool to room temperature, and then enter the stirred tank 2. Butanol, isopropanol and ethyl acetate are added to the tank 2 at room temperature, and stirred for 30 minutes. Finally, the products are packed by the packaging machine and shipped.

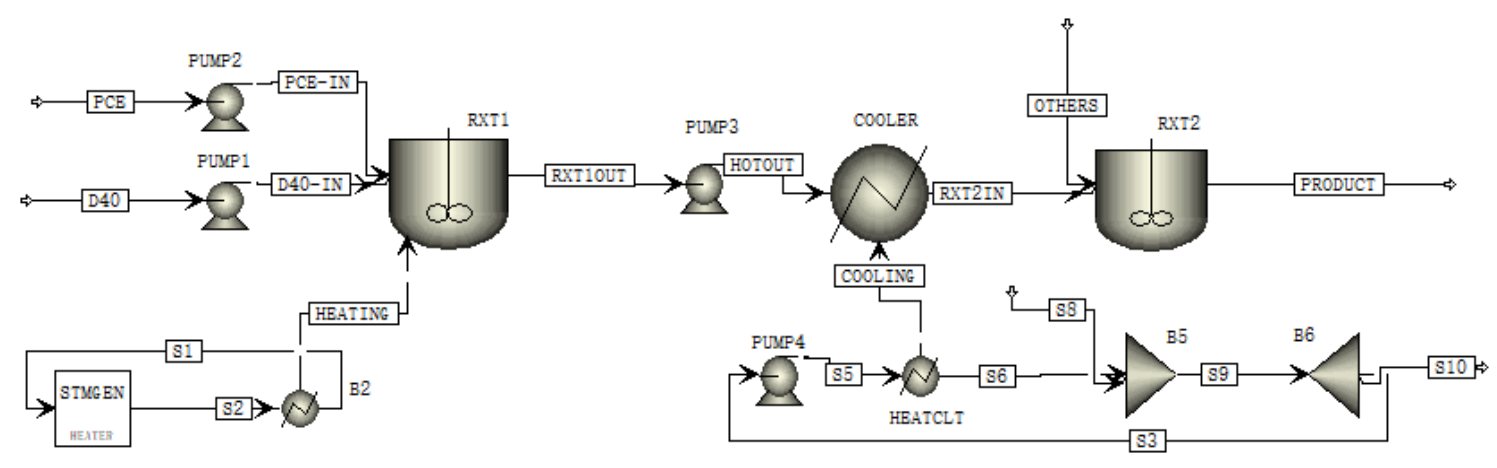

Figure 2. Electrified cleaning agent production process

\subsection{Electrified Cleaning Agent【Use】}

(1) Basic Process Information

Process name: electrified cleaning agent [use]

Process boundary: from gate to use

(2) Data representativeness

Main data source: estimation of the process parameters does not represent industry average data

Origin : China

Base year: 2020

Process equipment: air gun, spray gun, condensation recovery device

Main raw material: electrified cleaning fluid

Main energy consumption: electricity

Application scale: $2000 \mathrm{t} / \mathrm{y}$

Table 5. LCl data of the electrified cleaning agent 【use】 process

\begin{tabular}{|c|c|c|c|c|c|}
\hline Type & List name & Quantity & Unit & $\begin{array}{l}\text { Upstream } \\
\text { data source }\end{array}$ & $\begin{array}{l}\text { Use/emission } \\
\text { reason }\end{array}$ \\
\hline Energy & Electricity & 0.02 & MJ & $\begin{array}{l}\text { CLCD-China- } \\
\text { ECER } 0.8\end{array}$ & - \\
\hline $\begin{array}{l}\text { Environmental } \\
\text { emissions }\end{array}$ & Tetrachloroethylene & 0.06 & $\mathrm{~kg}$ & - & $\begin{array}{c}\text { Emissions } \\
\text { (atmosphere) }\end{array}$ \\
\hline $\begin{array}{l}\text { Environmental } \\
\text { emissions }\end{array}$ & $\begin{array}{l}\text { Naphtha (petroleum), } \\
\text { hydrotreated heavy }\end{array}$ & 0.13 & $\mathrm{~kg}$ & - & $\begin{array}{l}\text { Emissions } \\
\text { (atmosphere) }\end{array}$ \\
\hline $\begin{array}{l}\text { Environmental } \\
\text { emissions }\end{array}$ & Butanol & 0.01 & $\mathrm{~kg}$ & - & $\begin{array}{l}\text { Emissions } \\
\text { (atmosphere) }\end{array}$ \\
\hline $\begin{array}{l}\text { Environmental } \\
\text { emissions }\end{array}$ & Isopropanol & 0.02 & $\mathrm{~kg}$ & - & $\begin{array}{l}\text { Emissions } \\
\text { (atmosphere) }\end{array}$ \\
\hline $\begin{array}{c}\text { Environmental } \\
\text { emissions }\end{array}$ & Ethyl acetate & $3.62 \mathrm{E}-03$ & $\mathrm{~kg}$ & - & $\begin{array}{c}\text { Emissions } \\
\text { (atmosphere) }\end{array}$ \\
\hline
\end{tabular}


Technical supplementary description: First, the air gun is used to remove dust from the equipment. Then, the spray gun is used to spray the charged cleaning fluid to clean the equipment. Finally, the air gun is used to evaporate the cleaning fluid, while the recycling and condensing equipment is operated to recover the solvent. In the benchmark model, it is assumed that $80 \%$ of the solvent can be recycled, i.e., $20 \%$ of D40 solvent oil and tetrachloroethylene and all additives are discharged into the atmosphere.

\subsection{Electrified Cleaning Agent【Disposal】}

(1) Basic process information

Process name: electrified cleaning agent【disposal】

Process boundary: from use to grave

(2) Data representativeness

Main data sources: chemical process simulation, database. The results do not represent industry average data

Origin: China

Base year: 2020

Process equipment: distillation equipment, sludge landfill

Main raw material: Recycled electrified cleaning agent

Main energy consumption: electricity

Production scale: $2000 \mathrm{t} / \mathrm{y}$

Technical supplementary description: A two-stage distillation device is used to rectify the waste solution to separate tetrachloroethylene and D40 solvent oil. Figure 3 is a diagram of the two-stage distillation device for waste solvents simulated in ASPEN Plus. D40 solvent oil is simulated using a mixture of 50\% decane and 50\% undecane, and the sludge is simulated using ethyl palmitate. The used or unclean solvents in this process (i.e., detergents contain excessive soluble and non-volatile residues such as fatty acids, grease and oil) are first heated to above the boiling point of tetrachloroethylene $\left(121^{\circ} \mathrm{C}\right)$ to recycle high-purity tetrachloroethylene ( $99 \%$ purity). Then, the distillate from the bottom of the tower enters the second-stage distillation tower and is heated to the boiling point of D40 mineral spirits (greater than $190^{\circ} \mathrm{C}$ ) to volatilize and recover D40 mineral spirits. Residual impurities are left at the bottom of the kettle and become sludge due to their higher boiling point than D40 oil. Tetrachloroethylene and D40 solvent oil can be reused.

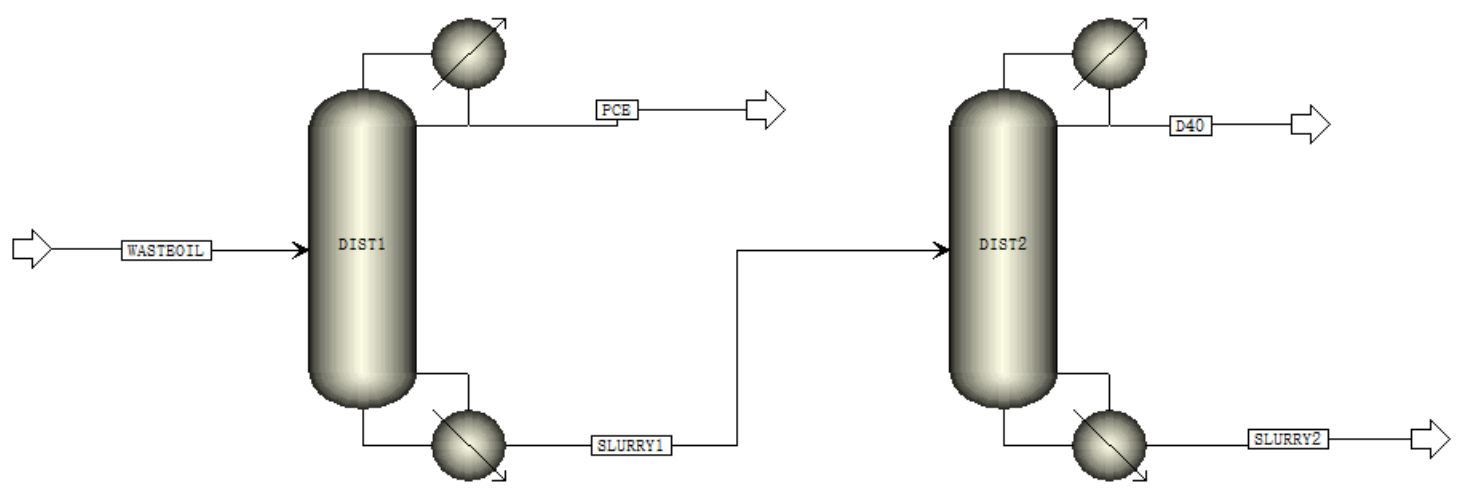

Figure 3. Design of a 2-stage distillation device for waste solvent 
Table 6. Calculation results of the 2-stage distillation unit for the distillation of the waste solvent (feed rate: $1000 \mathrm{~kg} / \mathrm{h}$ )

\begin{tabular}{ccc}
\hline Calculation results & Distillation Tower 1 (DIST1) & Distillation Tower 2 (DIST2) \\
\hline Minimum reflux ratio & 0.4581 & 0.0112 \\
Actual reflux ratio & 1.247 & 0.0527 \\
Minimum number of plates & 6.6628 & 2.4893 \\
Actual number of plates & 10 & 10 \\
Feed tray & 5.6298 & 5.5392 \\
Reboiler load/(MJ/h) & 489.12 & 200.44 \\
Condenser load/(MJ/h) & 161.08 & 207.1 \\
Distillate product & 120.95 & 182.17 \\
temperature & 190.91 & 275.68 \\
Bottom temperature & 0.3064 & 0.9635 \\
Distillate to feed ratio & & \\
\hline
\end{tabular}

Table 7. Material calculation results of the waste solvent 2-stage distillation unit

\begin{tabular}{|c|c|c|c|c|c|c|}
\hline $\begin{array}{l}\text { Calculation } \\
\text { results }\end{array}$ & Unit & $\begin{array}{r}\text { (WAST } \\
\text { EOIL })\end{array}$ & (PCE) & $\begin{array}{r}\text { （SLUR } \\
\text { RY1） }\end{array}$ & $\begin{array}{l}\text { Recovery } \\
\text { of solvent } \\
\text { oil (D40) }\end{array}$ & $\begin{array}{l}\text { (SLUR } \\
\text { RY2) }\end{array}$ \\
\hline Temperature & ${ }^{\circ} \mathrm{C}$ & 25 & 120.9547 & 190.9094 & 182.1664 & 275.6797 \\
\hline Pressure & bar & 1 & 1 & 1.2 & 1 & 1.2 \\
\hline Molar enthalpy & $\mathrm{cal} / \mathrm{mol}$ & -58436.548 & -9450.39 & -63022.5 & -60376.2 & -142478 \\
\hline Mass enthalpy & $\mathrm{cal} / \mathrm{gm}$ & -372.19905 & -57.0812 & -411.311 & -404.941 & -543.247 \\
\hline Molar entropy & $\begin{array}{c}\mathrm{cal} / \mathrm{mol}- \\
\mathrm{K}\end{array}$ & -203.01068 & -44.0808 & -229.724 & -226.883 & -334.24 \\
\hline Mass entropy & $\begin{array}{l}\mathrm{cal} / \mathrm{gm}- \\
\mathrm{K}\end{array}$ & -1.2930329 & -0.26625 & -1.49927 & -1.52169 & -1.27441 \\
\hline Molar density & $\mathrm{mol} / \mathrm{cc}$ & 0.0055618 & 0.008671 & 0.003912 & 0.004053 & 0.002421 \\
\hline Mass density & $\mathrm{gm} / \mathrm{cc}$ & 0.8732294 & 1.435496 & 0.599471 & 0.604314 & 0.634827 \\
\hline Enthalpy flow & $\mathrm{cal} / \mathrm{sec}$ & -107524.17 & -5327.89 & -80432 & -74246.2 & -6627.29 \\
\hline $\begin{array}{c}\text { Average } \\
\text { molecular weight }\end{array}$ & & 157.00349 & 165.5603 & 153.2235 & 149.0988 & 262.2706 \\
\hline Molar flow & $\mathrm{kmol} / \mathrm{hr}$ & 6.6240568 & 2.029588 & 4.594469 & 4.427016 & 0.167452 \\
\hline Mass Flow & $\mathrm{kg} / \mathrm{hr}$ & 1040 & 336.0192 & 703.9808 & 660.063 & 43.91784 \\
\hline D40 solvent oil & $\mathrm{kg} / \mathrm{hr}$ & 664 & 3.379192 & 660.6208 & 656.3036 & 4.317173 \\
\hline $\begin{array}{c}\text { Tetrachloroethyle } \\
\text { ne }\end{array}$ & $\mathrm{kg} / \mathrm{hr}$ & 336 & 332.64 & 3.36 & 3.359333 & 0.000667 \\
\hline $\begin{array}{c}\text { Sludge } \\
\text { Mass ratio } \\
\end{array}$ & $\mathrm{kg} / \mathrm{hr}$ & 40 & 0 & 40 & 0.4 & 39.6 \\
\hline D40 solvent oil & $\mathrm{wt} \%$ & 0.6384615 & 0.010057 & 0.938407 & 0.994305 & 0.098301 \\
\hline $\begin{array}{c}\text { Tetrachloroethyle } \\
\text { ne }\end{array}$ & $\mathrm{wt} \%$ & 0.3230769 & 0.989943 & 0.004773 & 0.005089 & $1.52 \mathrm{E}-05$ \\
\hline Sludge & $w t \%$ & 0.0384615 & 0 & 0.05682 & 0.000606 & 0.901684 \\
\hline Volume flow & $\mathrm{L} / \mathrm{min}$ & 19.849689 & 3.901314 & 19.57227 & 18.2042 & 1.153013 \\
\hline
\end{tabular}


Table 8. LCl data of electrified cleaning agent【Disposal】

\begin{tabular}{|c|c|c|c|c|c|}
\hline Type & List name & Quantity & Unit & $\begin{array}{c}\text { Upstream/downs } \\
\text { tream data } \\
\text { sources }\end{array}$ & $\begin{array}{l}\text { Use/emission } \\
\text { reason }\end{array}$ \\
\hline Energy & Electric power & 0.53 & MJ & $\begin{array}{l}\text { CLCD-China- } \\
\text { ECER } 0.8\end{array}$ & $\begin{array}{l}\text { Solvent } \\
\text { distillation } \\
\text { process }\end{array}$ \\
\hline $\begin{array}{l}\text { Waste to be } \\
\text { disposed of }\end{array}$ & Sludge & 0.04 & $\mathrm{~kg}$ & ELCD 3.0 & $\begin{array}{c}\text { Sludge landfill } \\
\text { after solvent } \\
\text { distillation }\end{array}$ \\
\hline Renewable waste & $\begin{array}{c}\text { Tetrachloroethyle } \\
\text { ne }\end{array}$ & 0.26 & $\mathrm{~kg}$ & & $\begin{array}{l}\text { Recovery and } \\
\text { reuse after solvent } \\
\text { distillation }\end{array}$ \\
\hline Renewable waste & Naphtha (D40) & 0.51 & $\mathrm{~kg}$ & & $\begin{array}{l}\text { Recovery and } \\
\text { reuse after solvent } \\
\text { distillation }\end{array}$ \\
\hline
\end{tabular}

\section{Life Cycle Impact Analysis}

\subsection{LCA Results}

The LCA results were modeled by the eFootprint software based on $1 \mathrm{~kg}$ electrified cleaning agent. The calculated indicators include global warming potential (GWP), acidification potential (AP), eutrophication potential (EP), respirable inorganic matter (RI), ozone depletion potential (ODP), photochemical ozone formation potential (POFP, also called POCP), ionizing radiation-human health potential (IRP), ecotoxicity (ET), human toxicity-carcinogenic (HT-cancer), and human toxicity-non-carcinogenic (HT-non cancer). The LCA results are as follows (Table 9).

Table 9. LCA results of electrified cleaning agent $(1 \mathrm{~kg})$

\begin{tabular}{ccc}
\hline Environmental impact indicators & $\begin{array}{c}\text { Impact type } \\
\text { indicator unit }\end{array}$ & LCA results \\
\hline GWP & $\mathrm{kg} \mathrm{CO}_{2}$ eq & 2.08 \\
AP & $\mathrm{kg} \mathrm{SO}_{2}$ eq & $9.49 \mathrm{E}-03$ \\
EP & $\mathrm{kg} \mathrm{PO}_{4}^{3-} \mathrm{eq}$ & $1.18 \mathrm{E}-03$ \\
RI & $\mathrm{kg} \mathrm{PM}_{2} .5 \mathrm{eq}$ & $2.13 \mathrm{E}-03$ \\
ODP & $\mathrm{kg} \mathrm{CFC}-11 \mathrm{eq}$ & $4.91 \mathrm{E}-05$ \\
POFP & $\mathrm{kg} \mathrm{NMVOC}$ eq & $2.89 \mathrm{E}-02$ \\
IRP & $\mathrm{kg} \mathrm{U} 235 \mathrm{eq}$ & $3.16 \mathrm{E}-02$ \\
ET & CTUe & $2.69 \mathrm{E}-01$ \\
HT-cancer & CTUh & $4.32 \mathrm{E}-08$ \\
HT-non cancer & CTUh & $2.31 \mathrm{E}-07$ \\
\hline
\end{tabular}

\subsection{Analysis of Process Cumulative Contribution}

The cumulative contribution of a process refers to the cumulative value of the direct contribution of the process and the contribution of all upstream processes (i.e., the contribution of raw material consumption). Since the process usually contains multiple inventory data, the process contribution analysis is actually the accumulation of the sensitivity of multiple inventory data. 
Table 10. Cumulative contribution results of electrified cleaning agent

\begin{tabular}{|c|c|c|c|c|c|}
\hline Name & GWP & AP & EP & $\mathbf{R I}$ & ODP \\
\hline $\begin{array}{c}\text { Electrified cleaning agent 【 } \\
\text { total】 }\end{array}$ & $2.08 \mathrm{E}+00$ & $9.49 \mathrm{E}-03$ & $1.18 \mathrm{E}-03$ & $2.13 \mathrm{E}-03$ & 4.91E-05 \\
\hline $\begin{array}{c}\text { Electrified cleaning agent 【 } \\
\text { production】 }\end{array}$ & $1.88 \mathrm{E}+00$ & $8.13 \mathrm{E}-03$ & $9.32 \mathrm{E}-04$ & $1.79 \mathrm{E}-03$ & 4.91E-05 \\
\hline electricity & $1.27 \mathrm{E}-02$ & 7.14E-05 & 4.44E-06 & $2.13 \mathrm{E}-05$ & $3.25 \mathrm{E}-11$ \\
\hline D40 solvent oil & 4.37E-01 & $1.95 \mathrm{E}-03$ & 2.79E-04 & $3.17 \mathrm{E}-04$ & $2.42 \mathrm{E}-07$ \\
\hline D40 solvent oil-transportation & $9.62 \mathrm{E}-03$ & $1.95 \mathrm{E}-04$ & $3.49 \mathrm{E}-05$ & $3.68 \mathrm{E}-05$ & $8.26 \mathrm{E}-10$ \\
\hline Tetrachloroethylene & $1.23 \mathrm{E}+00$ & $5.12 \mathrm{E}-03$ & $4.45 \mathrm{E}-04$ & $1.04 \mathrm{E}-03$ & $4.88 \mathrm{E}-05$ \\
\hline Tetrachloroethylene-Transport & 4.87E-03 & $9.86 \mathrm{E}-05$ & $1.77 \mathrm{E}-05$ & $1.86 \mathrm{E}-05$ & $4.18 \mathrm{E}-10$ \\
\hline Butanol & $3.62 \mathrm{E}-02$ & $1.79 \mathrm{E}-04$ & $5.10 \mathrm{E}-05$ & $3.12 \mathrm{E}-05$ & $4.05 \mathrm{E}-09$ \\
\hline Isopropanol & 8.98E-02 & $2.94 \mathrm{E}-04$ & $7.33 \mathrm{E}-05$ & $2.34 \mathrm{E}-04$ & $6.30 \mathrm{E}-09$ \\
\hline Ethyl acetate & 4.98E-03 & $3.55 \mathrm{E}-05$ & $7.58 \mathrm{E}-06$ & $5.64 \mathrm{E}-06$ & 2.09E-09 \\
\hline High-density polyethylene & $5.43 \mathrm{E}-02$ & $1.88 \mathrm{E}-04$ & $1.68 \mathrm{E}-05$ & $8.07 \mathrm{E}-05$ & $6.13 \mathrm{E}-09$ \\
\hline Electrified cleaning agent 【use & 1.99E-02 & $3.27 \mathrm{E}-04$ & $5.51 \mathrm{E}-05$ & $6.50 \mathrm{E}-05$ & $1.27 \mathrm{E}-09$ \\
\hline Direct contribution & $0.00 \mathrm{E}+00$ & $0.00 \mathrm{E}+00$ & $0.00 \mathrm{E}+00$ & $0.00 \mathrm{E}+00$ & $0.00 \mathrm{E}+00$ \\
\hline electricity & $5.28 \mathrm{E}-03$ & $2.98 \mathrm{E}-05$ & $1.85 \mathrm{E}-06$ & $8.88 \mathrm{E}-06$ & $1.35 \mathrm{E}-11$ \\
\hline Product transportation & $1.47 \mathrm{E}-02$ & $2.97 \mathrm{E}-04$ & 5.33E-05 & 5.61E-05 & $1.26 \mathrm{E}-09$ \\
\hline $\begin{array}{c}\text { Electrified cleaning agent 【 } \\
\text { Disposal】 }\end{array}$ & $1.86 \mathrm{E}-01$ & $1.04 \mathrm{E}-03$ & $1.89 \mathrm{E}-04$ & $2.84 \mathrm{E}-04$ & $1.45 \mathrm{E}-09$ \\
\hline electricity & $1.40 \mathrm{E}-01$ & 7.89E-04 & 4.90E-05 & $2.35 \mathrm{E}-04$ & $3.59 \mathrm{E}-10$ \\
\hline Sludge & $3.48 \mathrm{E}-02$ & $1.58 \mathrm{E}-05$ & $9.80 \mathrm{E}-05$ & $4.55 \mathrm{E}-06$ & $1.07 \mathrm{E}-10$ \\
\hline Sludge-Transport & $1.32 \mathrm{E}-04$ & $2.67 \mathrm{E}-06$ & 4.80E-07 & $5.05 \mathrm{E}-07$ & $1.13 \mathrm{E}-11$ \\
\hline Waste solvent-transportation & $1.13 \mathrm{E}-02$ & $2.29 \mathrm{E}-04$ & 4.10E-05 & 4.32E-05 & $9.70 \mathrm{E}-10$ \\
\hline
\end{tabular}

Table 10. Cumulative contribution results of electrified cleaning agent (continued)

\begin{tabular}{|c|c|c|c|c|c|}
\hline Name & POFP & IRP & ET & HT-cancer & $\begin{array}{c}\text { HT-non } \\
\text { cancer }\end{array}$ \\
\hline $\begin{array}{c}\text { Electrified cleaning agent 【 } \\
\text { total】] }\end{array}$ & 2.89E-02 & 3.16E-02 & 2.69E-01 & 4.32E-08 & 2.31E-07 \\
\hline $\begin{array}{c}\text { Electrified cleaning agent 【 } \\
\text { production】 }\end{array}$ & 6.36E-03 & 2.26E-02 & 2.21E-01 & 2.19E-08 & $1.51 \mathrm{E}-07$ \\
\hline electricity & 5.27E-06 & 7.63E-04 & 6.08E-05 & 3.32E-12 & $7.86 \mathrm{E}-12$ \\
\hline D40 solvent oil & 1.77E-03 & $1.48 \mathrm{E}-02$ & 1.00E-01 & 4.82E-09 & 7.88E-09 \\
\hline $\begin{array}{l}\text { D40 solvent oil- } \\
\text { transportation }\end{array}$ & 5.87E-05 & 5.16E-05 & 3.94E-04 & $1.65 \mathrm{E}-11$ & 2.69E-11 \\
\hline Tetrachloroethylene & 3.92E-03 & 2.07E-04 & $1.08 \mathrm{E}-01$ & 1.66E-08 & $1.40 \mathrm{E}-07$ \\
\hline $\begin{array}{c}\text { Tetrachloroethylene- } \\
\text { Transport }\end{array}$ & 2.97E-05 & 2.61E-05 & 1.99E-04 & 8.33E-12 & $1.36 \mathrm{E}-11$ \\
\hline Butanol & 1.44E-04 & 4.19E-03 & $5.12 E-03$ & $1.44 \mathrm{E}-10$ & $1.41 \mathrm{E}-09$ \\
\hline Isopropanol & 1.34E-04 & 7.16E-04 & $3.03 E-03$ & $1.29 \mathrm{E}-10$ & $2.11 \mathrm{E}-10$ \\
\hline Ethyl acetate & 2.26E-05 & 9.49E-04 & $1.25 E-03$ & $2.27 \mathrm{E}-11$ & $3.21 \mathrm{E}-10$ \\
\hline High-density polyethylene & $2.81 \mathrm{E}-04$ & 8.72E-04 & $2.65 \mathrm{E}-03$ & $1.26 \mathrm{E}-10$ & $2.06 \mathrm{E}-10$ \\
\hline $\begin{array}{c}\text { Electrified cleaning agent 【 } \\
\text { use】 }\end{array}$ & 2.24E-02 & 3.97E-04 & 4.76E-02 & $2.12 \mathrm{E}-08$ & 8.01E-08 \\
\hline Direct contribution & 2.23E-02 & 0 & 4.70E-02 & 2.12E-08 & 8.00E-08 \\
\hline electricity & $2.20 \mathrm{E}-06$ & 3.18E-04 & 2.53E-05 & $1.38 \mathrm{E}-12$ & $3.27 E-12$ \\
\hline Product transportation & 8.96E-05 & 7.87E-05 & $6.00 \mathrm{E}-04$ & $2.51 \mathrm{E}-11$ & 4.10E-11 \\
\hline
\end{tabular}




\begin{tabular}{ccccccc}
$\begin{array}{c}\text { Electrified cleaning agent } \\
\text { Disposal】 } \\
\text { electricity }\end{array}$ & $1.47 \mathrm{E}-04$ & $8.62 \mathrm{E}-03$ & $1.24 \mathrm{E}-03$ & $5.67 \mathrm{E}-11$ & $1.37 \mathrm{E}-10$ \\
$\begin{array}{c}\text { Sludge } \\
\text { Sludge-Transport }\end{array}$ & $5.82 \mathrm{E}-05$ & $8.43 \mathrm{E}-03$ & $6.71 \mathrm{E}-04$ & $3.67 \mathrm{E}-11$ & $8.68 \mathrm{E}-11$ \\
Waste solvent-transportation & $8.06 \mathrm{E}-05$ & $1.29 \mathrm{E}-04$ & $1.04 \mathrm{E}-04$ & $4.36 \mathrm{E}-13$ & $1.86 \mathrm{E}-11$ \\
\hline & $7.08 \mathrm{E}-07$ & $5.40 \mathrm{E}-06$ & $2.26 \mathrm{E}-13$ & $3.69 \mathrm{E}-13$ \\
& $6.06 \mathrm{E}-05$ & $4.62 \mathrm{E}-04$ & $1.93 \mathrm{E}-11$ & $3.16 \mathrm{E}-11$ \\
\hline
\end{tabular}

According to the LCA results of electrified cleaning agent【 production 】 (Figures 4 - 13), the results of various indicators are as follows:

(1) For the GWP indicator, the three processes that contribute the most are the production of tetrachloroethylene $\left(1.23 \mathrm{~kg} \mathrm{CO}_{2} \mathrm{eq}\right)$, the production of D40 solvent oil $\left(0.437 \mathrm{~kg} \mathrm{CO}_{2}\right.$ eq), and electricity (electrified cleaning agent 【disposal】) $(0.14$ $\mathrm{kg} \mathrm{CO}$ ) eq).

(2) Acidification (AP): The three processes that contribute the most are the production of tetrachloroethylene (5.12E-03 $\mathrm{kg} \mathrm{SO}_{2}$ eq), the production of D40 solvent oil (1.95E$03 \mathrm{~kg} \mathrm{SO}_{2}$ eq), and electricity (electrified cleaning agent【disposal】) (7.89E-04 kg $\mathrm{SO} 2 \mathrm{eq})$.

(3) Eutrophication potential (EP): The three most contributing processes are tetrachloroethylene production (4.45E-04 $\left.\mathrm{kg} \mathrm{PO}_{4}{ }^{3-} \mathrm{eq}\right), \mathrm{D} 40$ solvent oil production (2.79E-04 $\mathrm{kg} \mathrm{PO}_{4}{ }^{3-} \mathrm{eq}$ ), and sludge landfill (7.89E-04 $\left.\mathrm{kg} \mathrm{PO}_{4}{ }^{3-} \mathrm{eq}\right)$.

(4) Inhalable inorganic matter (RI): The four processes that contribute the most are tetrachloroethylene production (1.04E-03 kg PM2.5 eq), D40 solvent oil production (3.17E-04 kg PM2.5 eq), electricity (electrified cleaning agent 【disposal】) (2.35E$04 \mathrm{~kg}$ PM2.5 eq), and isopropanol production (2.34E-04 kg PM2.5 eq).

(5) Ozone depletion potential (ODP): The three processes that contribute the most are tetrachloroethylene production (4.88E-05 kg CFC-11 eq), D40 solvent oil production (2.42E-07 kg CFC-11 eq), and isopropanol production (6.30E-09 kg CFC-11 eq).

(6) Photochemical ozone formation potential (POFP): The three processes that contribute the most are the environmental emissions caused by solvent volatilization during the process of electrified cleaning agent【use】(2.23E-02 kg NMVOC eq), the production of tetrachloroethylene (3.92E-03 kg NMVOC eq) ), and D40 solvent oil production (1.77E-03 kg NMVOC eq).

(7) Ionizing radiation-Human Health (IRP): The three processes that contribute the most are D40 solvent oil production $(1.48 \mathrm{E}-02 \mathrm{~kg} \mathrm{U} 235 \mathrm{eq}$ ), electricity (electrified cleaning agent 【Disposal】) (8.43E-03 kg U235 eq), and D40 production (4.19E-03 $\mathrm{kg} \mathrm{U} 235$ eq).

(8) Ecotoxicity (ET): The three processes that contribute the most are the production of tetrachloroethylene (1.08E-01 CTUe), the production of D40 solvent oil (1.00E-01 CTUe), and the environment emissions caused by the volatilization of solvents in the process of electrified cleaning agent【use】 (4.70E-02 CTUe).

(9) Human toxicity-carcinogenic (HT-cancer): The three processes that contribute the most are the environmental emissions (2.12E-08 CTUh) caused by solvent volatilization during the process of the charged cleaner 【use】, the production of tetrachloroethylene (1.66E-08 CTUh), and D40 solvent oil production (4.82E-09 CTUh).

(10)Human toxicity-non-carcinogenic (HT-non cancer): The three processes that contribute the most are the production of tetrachloroethylene (1.40E-07 CTUh), the 
environmental emissions caused by solvent volatilization during the process of electrified cleaning agent【use】(8.00E-08). CTUh), and D40 solvent oil production (7.88E-09 CTUh).

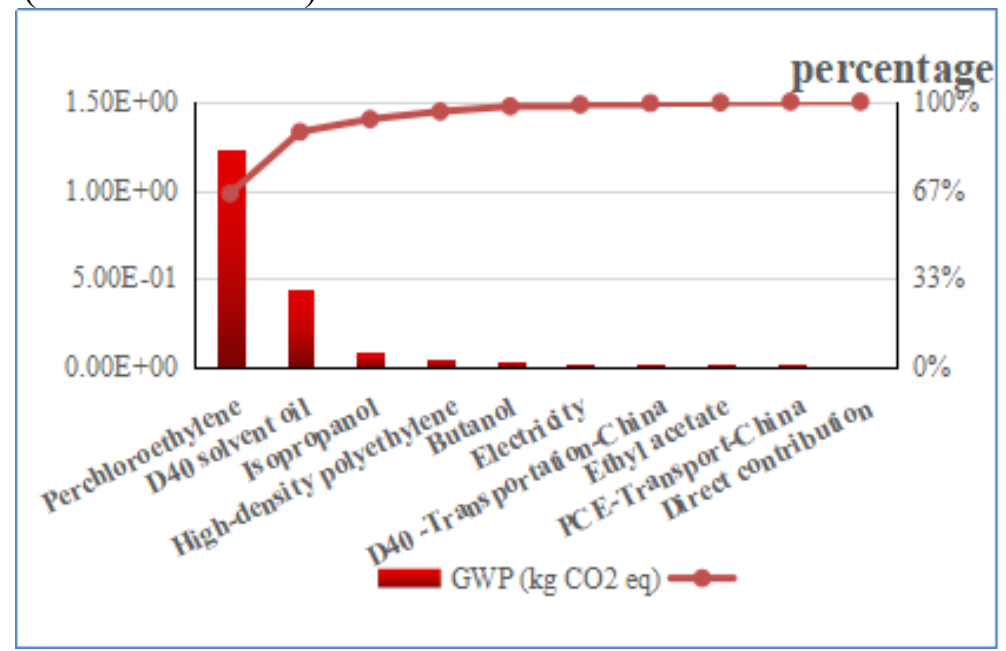

Figure 4. Global warming potential (GWP) results of electrified cleaning agent 【production】

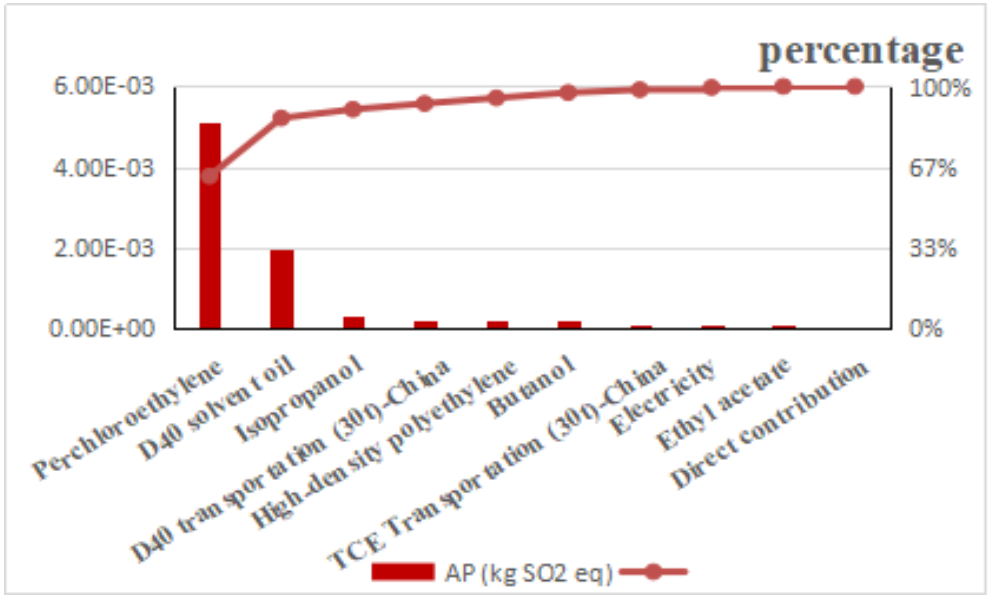

Figure 5. Acidification potential (AP) results of electrified cleaning agent【production】

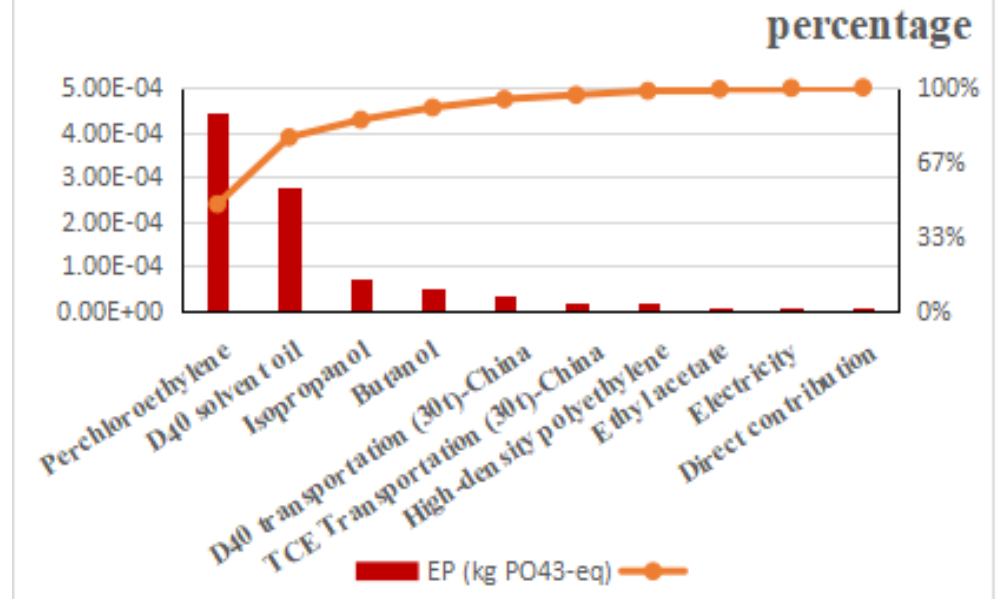

Figure 6. The eutrophication potential (EP) results of electrified cleaning agent 【production】 


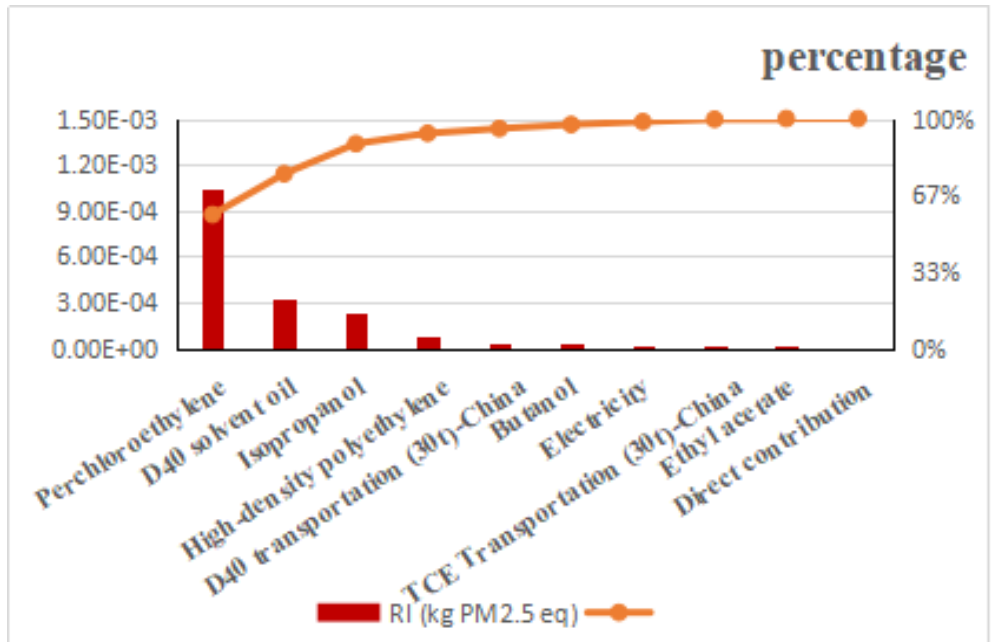

Figure 7. Inhalable inorganic matter (RI) results of electrified cleaning agent 【production】

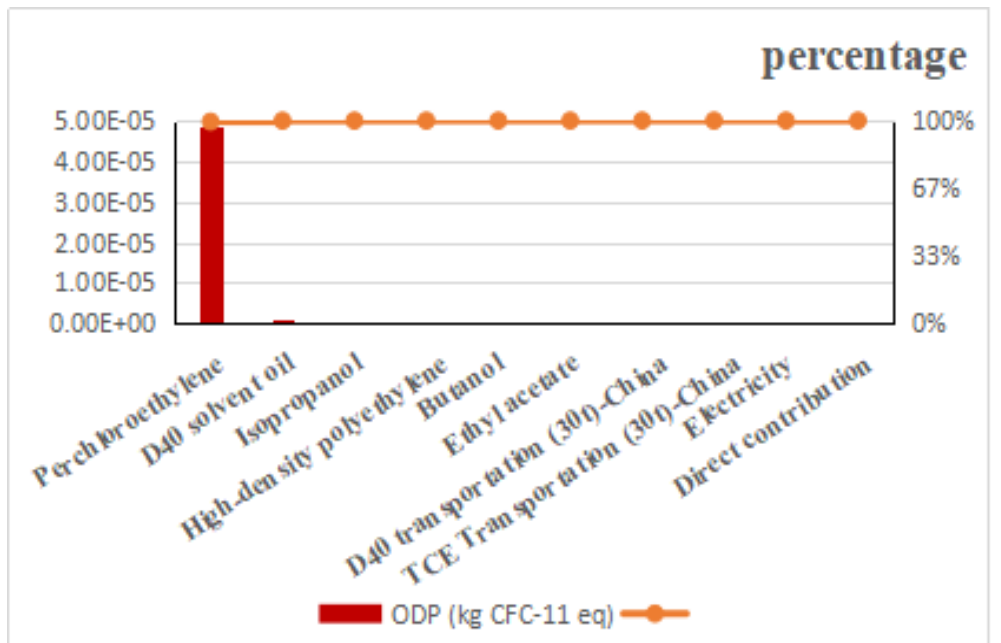

Figure 8. Ozone depletion (ODP) results of electrified cleaning agent 【production】

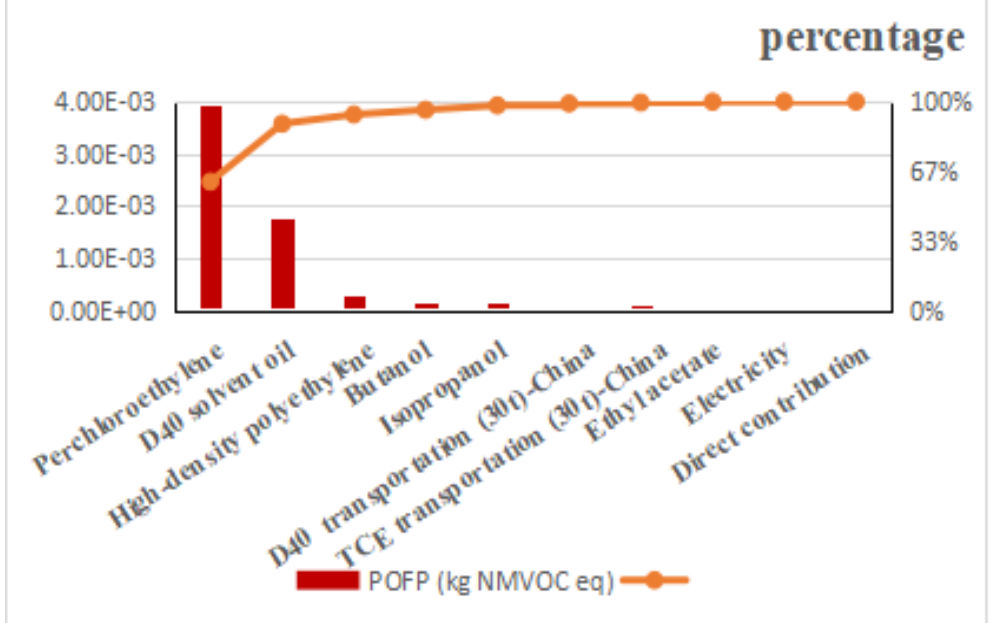

Figure 9. Photochemical ozone formation potential (POFP) results of electrified cleaning agent 【 production】 


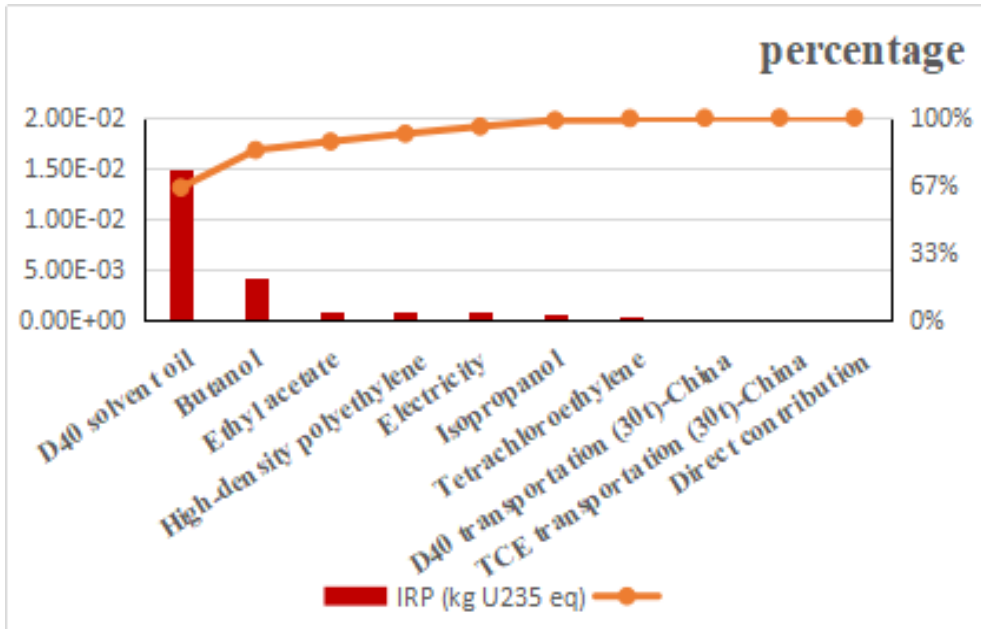

Figure 10. lonizing radiation potential (IRP) results of electrified cleaning agent (production】

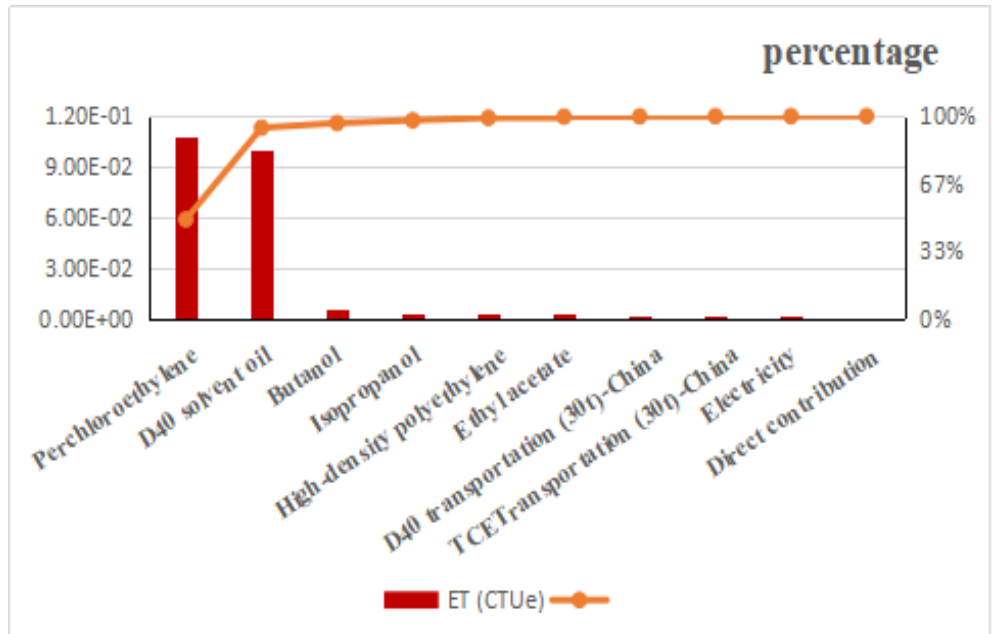

Figure 11. Ecotoxicity $(E T)$ results of electrified cleaning agent 【production】

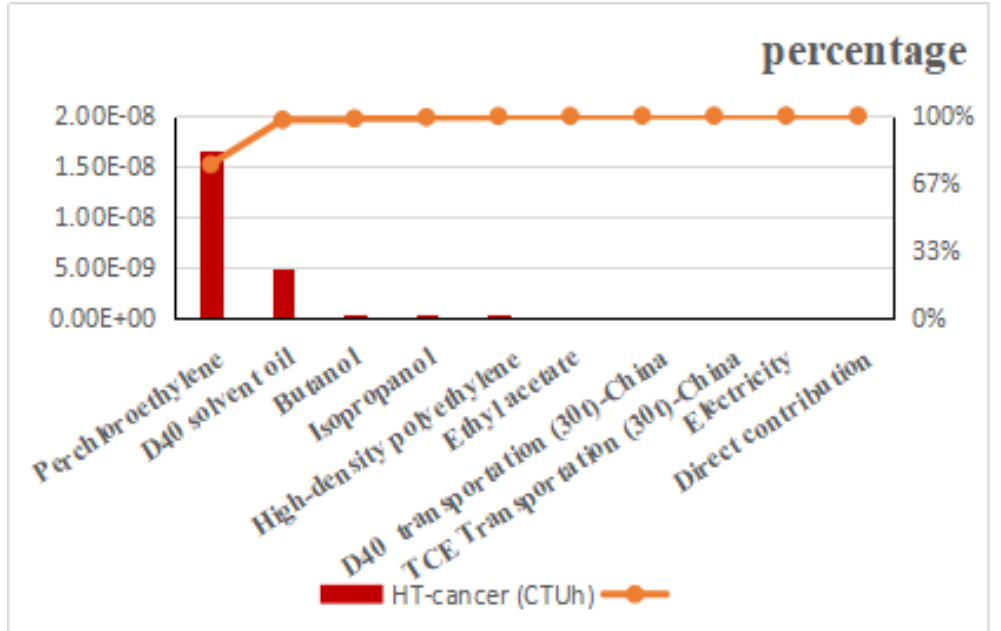

Figure 12. Human toxicity-carcinogenic (HT-cancer) results of electrified cleaning agent 【 production】 


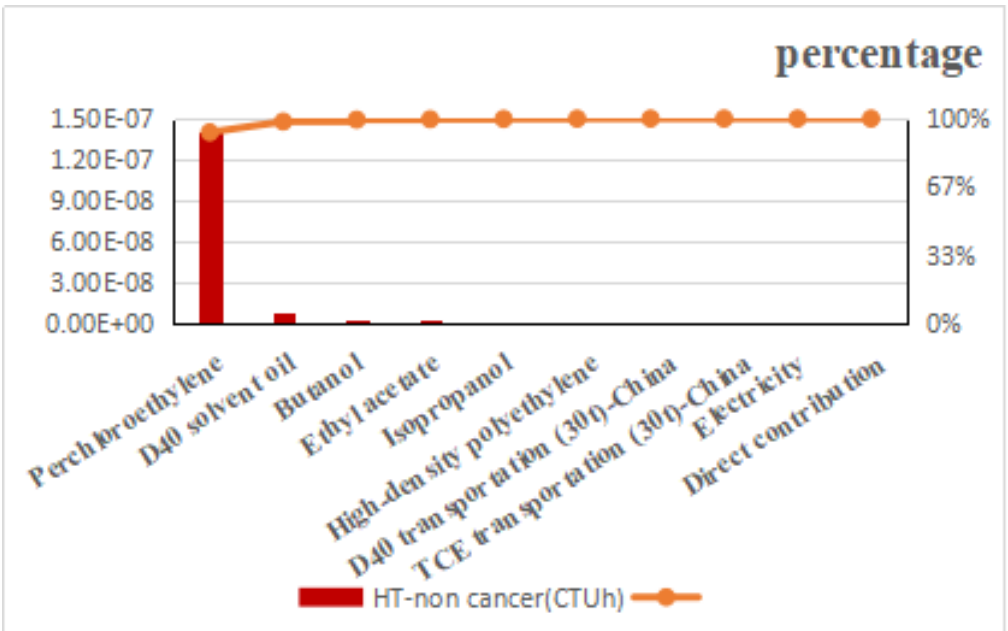

Figure 13. Human toxicity-HT-non cancer results of electrified cleaning agent 【production】

\subsection{Sensitivity Analysis of Inventory Data}

Inventory data sensitivity refers to the corresponding indicator change rate caused by the unit change rate of inventory data. By analyzing the sensitivity of the inventory data to each indicator and cooperating with the improvement potential evaluation, the most effective improvement points can be identified. Table 11 lists the inventory data with sensitivity $>0.5 \%$.

According to the average sensitivity (contribution rate) analysis:

(1) The five processes with the highest average sensitivity are tetrachloroethylene production $51.48 \%$, D40 solvent oil production $12.66 \%$, tetrachloroethylene environmental emissions 【use】 $11.85 \%$, butanol emissions 【use】 5.54\%, and electricity【disposal】 $3.82 \%$.

(2) For the GWP, the top 5 processes with the highest sensitivity are tetrachloroethylene production $58.95 \%$, D40 solvent oil production $20.96 \%$, electricity 【Disposal】 $6.71 \%$, isopropanol production $4.31 \%$, and high-density polyethylene $2.61 \%$.

(3) For the AP, the top five processes with the highest sensitivity are tetrachloroethylene production $53.90 \%$, D40 solvent oil production $20.57 \%$, electricity 【Disposal】 $8.31 \%$, product transportation $3.13 \%$, and isopropanol production $3.10 \%$.

(4) For the EP, the top 5 processes with the highest sensitivity are tetrachloroethylene production $37.84 \%$, D40 solvent oil production $23.73 \%$, sludge landfill $8.34 \%$, isopropanol production $6.24 \%$, and product transportation $4.53 \%$.

(5) For the RI, the top 5 processes with the highest sensitivity are tetrachloroethylene production $48.77 \%$, D40 solvent oil production $14.86 \%$, electricity 【Disposal】 $11.02 \%$, isopropanol production $10.94 \%$, and high-density polyethylene $3.78 \%$.

(6) For ozone depletion (ODP), the most sensitive processes are tetrachloroethylene production (99.46\%) and the production of D40 solvent oil $(0.49 \%)$.

(7) For the POFP, the most sensitive processes are $44.31 \%$ of butanol environmental emissions, $17.37 \%$ of isopropanol environmental emissions, $13.57 \%$ of tetrachloroethylene production, $11.03 \%$ of tetrachloroethylene environmental emissions, and $6.12 \%$ of D40 solvent oil production. 
Table 11. Sensitivity of inventory data (unit: \%)

\begin{tabular}{|c|c|c|c|c|c|c|c|c|c|c|c|}
\hline Process & GWP & $\mathbf{A P}$ & EP & RI & ODP & POFP & IRP & ET & $\begin{array}{c}\text { HT- } \\
\text { canc } \\
\text { er }\end{array}$ & $\begin{array}{c}\text { HT- } \\
\text { non } \\
\text { canc } \\
\text { er }\end{array}$ & $\begin{array}{l}\text { Aver } \\
\text { age } \\
\text { sensi } \\
\text { tivity }\end{array}$ \\
\hline $\begin{array}{l}\text { Electrified cleaning } \\
\text { agent 【production】 }\end{array}$ & 90.1 & 85.64 & 79.26 & 83.67 & 99.99 & 22.03 & 71.45 & 81.85 & 50.71 & 65.23 & 72.08 \\
\hline $\begin{array}{l}\text { Electrified cleaning } \\
\text { agent【use】 }\end{array}$ & 0.96 & 3.44 & 4.692 & 3.044 & 0.003 & 77.458 & 1.256 & 17.68 & 49.15 & 34.70 & 21.68 \\
\hline $\begin{array}{l}\text { Electrified cleaning } \\
\text { agent【disposal】 }\end{array}$ & 8.93 & 10.91 & 16.043 & 13.284 & 0.003 & 0.508 & $\begin{array}{c}27.29 \\
0\end{array}$ & 0.461 & 0.131 & 0.060 & 6.23 \\
\hline \multicolumn{12}{|l|}{$\begin{array}{l}\text { Electrified cleaning } \\
\text { agent【production】 }\end{array}$} \\
\hline Electricity & 0.61 & 0.75 & 0.377 & 0.998 & 0.000 & 0.018 & 2.417 & 0.023 & 0.008 & 0.003 & 0.35 \\
\hline D40 solvent oil & 20.96 & 20.56 & 23.728 & 14.86 & 0.494 & 6.122 & $\begin{array}{c}46.84 \\
2\end{array}$ & 37.22 & 11.176 & 3.414 & 12.66 \\
\hline $\begin{array}{l}\text { D40 solvent Oil- } \\
\text { transportation }\end{array}$ & 0.46 & 2.05 & 2.974 & 1.723 & 0.002 & 0.203 & 0.163 & 0.146 & 0.038 & 0.012 & 0.93 \\
\hline Tetrachloroethylene & 58.95 & 53.89 & 37.844 & 48.771 & 99.46 & 13.573 & 0.656 & 39.91 & 38.502 & 60.86 & 51.48 \\
\hline $\begin{array}{c}\text { Tetrachloroethylene- } \\
\text { Transport }\end{array}$ & 0.23 & 1.04 & 1.505 & 0.872 & 0.001 & 0.103 & 0.083 & 0.074 & 0.019 & 0.006 & 0.47 \\
\hline Butanol & 1.74 & 1.88 & 4.335 & 1.463 & 0.008 & 0.499 & $\begin{array}{c}13.25 \\
7\end{array}$ & 1.902 & 0.334 & 0.613 & 1.36 \\
\hline Isopropanol & 4.31 & 3.09 & 6.235 & 10.940 & 0.013 & 0.464 & 2.268 & 1.125 & 0.299 & 0.091 & 3.18 \\
\hline Ethyl acetate & 0.239 & 0.37 & 0.645 & 0.264 & 0.004 & 0.078 & 3.007 & 0.465 & 0.052 & 0.139 & 0.22 \\
\hline $\begin{array}{l}\text { High-density } \\
\text { Polyethylene }\end{array}$ & 2.61 & 1.98 & 1.433 & 3.781 & 0.012 & 0.973 & 2.761 & 0.985 & 0.291 & 0.089 & 1.40 \\
\hline \multicolumn{12}{|l|}{$\begin{array}{l}\text { Electrified cleaning } \\
\text { agent【 【use】 }\end{array}$} \\
\hline Electricity & 0.25 & 0.31 & 0.157 & 0.416 & 0.000 & 0.008 & 1.007 & 0.009 & 0.003 & 0.001 & 0.14 \\
\hline Product Transportation & 0.70 & 3.13 & 4.535 & 2.628 & 0.003 & 0.310 & 0.249 & 0.223 & 0.058 & 0.018 & 1.42 \\
\hline Tetrachloroethylene & 0.000 & 0.000 & 0.000 & 0.000 & 0.000 & 11.032 & 0.000 & 17.45 & 49.088 & 34.68 & 11.85 \\
\hline Butanol & 0.0 & 0.0 & 0.0 & 0.0 & 0.0 & 44.31 & 0.0 & 0.0 & 0.0 & 0.0 & 5.54 \\
\hline Isopropanol & 0.0 & 0.0 & 0.0 & 0.0 & 0.0 & 17.37 & 0.0 & 0.0 & 0.0 & 0.0 & 2.17 \\
\hline Ethyl acetate & 0.0 & 0.0 & 0.0 & 0.0 & 0.0 & 4.43 & 0.0 & 0.0 & 0.0 & 0.0 & 0.55 \\
\hline \multicolumn{12}{|l|}{$\begin{array}{l}\text { Electrified cleaning } \\
\text { agent【disposal】 }\end{array}$} \\
\hline Electricity & 6.71 & 8.31 & 4.168 & 11.024 & 0.001 & 0.202 & $\begin{array}{c}26.68 \\
7\end{array}$ & 0.249 & 0.085 & 0.038 & 3.82 \\
\hline Sludge & 1.67 & 0.17 & 8.342 & 0.213 & 0.000 & 0.064 & $\begin{array}{c}0.40 \\
9\end{array}$ & 0.038 & 0.001 & 0.008 & 1.31 \\
\hline Sludge-Transport Waste & 0.006 & 0.028 & 0.041 & 0.024 & 0.000 & 0.003 & $\begin{array}{c}0.00 \\
2\end{array}$ & 0.002 & 0.001 & 0.000 & 0.01 \\
\hline $\begin{array}{l}\text { Solvent-vehicle } \\
\text { Transportation }\end{array}$ & 0.54 & 2.41 & 3.492 & 2.023 & 0.002 & 0.239 & $\begin{array}{c}0.19 \\
2\end{array}$ & 0.172 & 0.045 & 0.014 & 1.10 \\
\hline
\end{tabular}

(8) For the IRP, the most sensitive processes are D40 solvent oil production $(46.84 \%)$, electricity【Disposal】(26.69\%), and butanol production (13.26\%).

(9) For the eco-toxicity (ET), the most sensitive processes are $39.91 \%$ of tetrachloroethylene production, $37.22 \%$ of D40 solvent oil production, and $17.45 \%$ of environmental emissions of tetrachloroethylene 【use】.

(10)For Human toxicity-carcinogenic (HT-cancer), the most sensitive processes are tetrachloroethylene environmental emission【use 】 $49.09 \%$, tetrachloroethylene production $38.50 \%$, and D40 solvent oil production $11.18 \%$. 
(11)For Human toxicity-HT-non cancer, the most sensitive processes are tetrachloroethylene production $60.86 \%$, tetrachloroethylene environmental emissions

【use】 $34.69 \%$, and D40 solvent oil production $3.41 \%$.

Comprehensive analysis shows that the four processes including tetrachloroethylene production, D40 solvent oil production, environmental discharge of tetrachloroethylene【use】 and electricity【product disposal】 have significant effects on each LCA indicator. So, they are the focus of process improvement. The environmental emission of butanol only affects the photochemical ozone formation potential (POFP).

4.4 Possible Process Improvement

(1) Tetrachloroethylene and D40 solvent oil are the necessary active ingredients in electrified cleaner products. It is recommended to optimize the production process to reduce environmental emissions, or to develop a cleaner production process.

(2) Efforts should be made to reduce the environmental emissions of tetrachloroethylene and other solvents during the use of the product, develop the efficient solvent recovery equipment, and improve operating procedures.

(3) Distillation for the recovery of waste solvents is a process with high energy consumption. Renewable energy should be used to replace electricity as much as possible to obtain carbon credits.

\subsection{The Impact of Electricity Consumption during Production on Carbon Footprint}

In the original model, the energy consumption of heating, stirring, cooling, and material transportation during the production process is considered. The power consumption estimated is $0.05 \mathrm{MJ}$. The simulated production process did not consider other processes that may be used, such as the energy consumption of the filtration process. It is estimated that all production energy consumption should be less than 0.1 MJ. Table 15 shows the impact of increasing power consumption from $0.05 \mathrm{MJ}$ to $1 \mathrm{MJ}$ on total carbon emissions. It can be seen that when the energy consumption increases to $0.1 \mathrm{MJ}$, the total carbon emissions only increase by $0.96 \%$. When the electricity consumption further increases 10 times to $1 \mathrm{MJ}$, carbon emissions will increase by about $12 \%$. Therefore, under the premise of paying attention to energy conservation in the production process, electricity consumption has little effect on the carbon footprint.

Table 15. Impact of electricity consumption on carbon footprint

\begin{tabular}{cccc}
\hline $\begin{array}{c}\text { Power } \\
\text { consumption /MJ }\end{array}$ & $\begin{array}{c}\text { Production process GWP } \\
/(\mathbf{k g ~ C O} \mathbf{~} \mathbf{~ e q})\end{array}$ & $\begin{array}{c}\text { Total carbon emissions } \\
\text { GWP /(kg CO } \mathbf{~ e q ) ~}\end{array}$ & $\begin{array}{c}\text { Increase in } \\
\text { carbon } \\
\text { emissions /\% }\end{array}$ \\
\hline $\mathbf{0 . 0 5 ( B e n c h m a r k )}$ & 1.88 & 2.08 & - \\
$\mathbf{0 . 1}$ & 1.89 & 2.10 & +0.96 \\
$\mathbf{0 . 5}$ & 2.00 & 2.20 & +5.77 \\
$\mathbf{1}$ & 2.13 & 2.33 & +12.02 \\
\hline
\end{tabular}

\subsection{Impact of Transportation Distance on Carbon Footprint}

In the original model, only the transportation of D40 solvent oil and tetrachloroethylene, which accounted for $64 \%$ and $33 \%$ of the components, respectively, was considered. The transportation distance was assumed to be $200 \mathrm{~km}$, which is close to 
the national average goods transportation distance of $187 \mathrm{~km}$. The data in Table 16 shows that increasing the transportation distance of D40 solvent oil to $2000 \mathrm{~km}$ will only increase the total carbon emissions by $4.33 \%$. Therefore, the transportation distance of raw materials has little effect on total carbon emissions.

Table 16. The impact of D40 solvent oil transportation distance on carbon footprint

\begin{tabular}{cccc}
\hline $\begin{array}{c}\text { Transport } \\
\text { distance } / \mathbf{k m}\end{array}$ & $\begin{array}{c}\text { Transport GWP } /(\mathbf{k g} \\
\left.\mathbf{C O}_{\mathbf{2}} \mathbf{~ e q}\right)\end{array}$ & $\begin{array}{c}\text { Total carbon emissions } \\
\mathbf{G W P} /(\mathbf{k g ~ C O} \mathbf{~ e q})\end{array}$ & $\begin{array}{c}\text { Increase in } \\
\text { carbon } \\
\text { emissions } / \%\end{array}$ \\
\hline $\mathbf{2 0 0}$ (Benchmark) & $9.62 \mathrm{E}-03$ & 2.08 & - \\
$\mathbf{5 0 0}$ & $2.40 \mathrm{E}-02$ & 2.10 & +0.40 \\
$\mathbf{1 0 0 0}$ & $4.81 \mathrm{E}-02$ & 2.12 & +1.92 \\
$\mathbf{2 0 0 0}$ & $9.62 \mathrm{E}-02$ & 2.17 & +4.33 \\
\hline
\end{tabular}

In the original model, the product transport distance is $200 \mathrm{~km}$. The data in Table 17 shows that when the product transportation distance is increased to $2000 \mathrm{~km}$, the total carbon emissions only increase by $6.73 \%$. Therefore, product transportation distance has little effect on total carbon emissions.

Table 17. Impact of product transportation distance on carbon footprint

\begin{tabular}{cccc}
\hline $\begin{array}{c}\text { Transport } \\
\text { distance } / \mathbf{k m}\end{array}$ & $\begin{array}{c}\text { Transport GWP } /(\mathbf{k g} \\
\left.\mathbf{C O}_{\mathbf{2}} \mathbf{~ e q}\right)\end{array}$ & $\begin{array}{c}\text { Total carbon emissions GWP } \\
/(\mathbf{k g ~ C O} \mathbf{~} \mathbf{~ e q})\end{array}$ & $\begin{array}{c}\text { Increase in } \\
\text { carbon } \\
\text { emissions } / \%\end{array}$ \\
\hline $\mathbf{2 0 0}$ (Benchmark) & $9.62 \mathrm{E}-03$ & 2.08 & - \\
$\mathbf{5 0 0}$ & $2.40 \mathrm{E}-02$ & 2.10 & +0.40 \\
$\mathbf{1 0 0 0}$ & $4.81 \mathrm{E}-02$ & 2.12 & +1.92 \\
$\mathbf{2 0 0 0}$ & $9.62 \mathrm{E}-02$ & 2.17 & +4.33 \\
\hline
\end{tabular}

\subsection{Comparison with LCA Indicators of Other Electrified Cleaner Production Processes}

The LCA results of this study is further compared with the LCA indicators of other three common electrified cleaner production processes, including trichloroethylene, tetrachloroethylene and n-bromopropane (data source is the Ecoinvent 3.1 database).

Table 18. Comparison of LCA indicators in the production process of electrified cleaning agent

\begin{tabular}{cccccc}
\hline Name & GWP & AP & EP & RI & ODP \\
\hline Electrified cleaning & $1.88 \mathrm{E}+00$ & $8.13 \mathrm{E}-03$ & $9.32 \mathrm{E}-04$ & $1.79 \mathrm{E}-03$ & $4.91 \mathrm{E}-05$ \\
agent 【production】 & $4.92 \mathrm{E}-01$ & $3.32 \mathrm{E}-02$ & $2.01 \mathrm{E}-03$ & $5.29 \mathrm{E}-03$ & $4.00 \mathrm{E}-05$ \\
Trichloroethylene & -73.81 & 308.17 & 115.98 & 195.91 & -18.49 \\
Change ratio (\%) & $3.83 \mathrm{E}+00$ & $1.62 \mathrm{E}-02$ & $1.46 \mathrm{E}-03$ & $3.30 \mathrm{E}-03$ & $1.50 \mathrm{E}-04$ \\
Tetrachloroethylene & 103.82 & 99.11 & 56.22 & 84.53 & 205.62 \\
Change ratio (\%) & $5.23 \mathrm{E}+00$ & $2.62 \mathrm{E}-02$ & $6.63 \mathrm{E}-03$ & $4.41 \mathrm{E}-03$ & $1.15 \mathrm{E}-06$ \\
N-bromopropane & 178.74 & 222.15 & 611.74 & 146.78 & -97.65 \\
Change ratio (\%) & & & & & \\
\hline
\end{tabular}


Table 18. Comparison of LCA indicators in the production process of electrified cleaning agent (continued)

\begin{tabular}{cccccc}
\hline Name & POFP & IRP & ET & HT-cancer & HT-non cancer \\
\hline Electrified cleaning & $6.36 \mathrm{E}-03$ & $2.26 \mathrm{E}-02$ & $2.21 \mathrm{E}-01$ & $2.19 \mathrm{E}-08$ & $1.51 \mathrm{E}-07$ \\
agent 【production 】 & & & & \\
Trichloroethylene & $1.91 \mathrm{E}-02$ & $6.36 \mathrm{E}-03$ & $4.47 \mathrm{E}-01$ & $8.74 \mathrm{E}-08$ & $4.44 \mathrm{E}-07$ \\
Change ratio (\%) & 200.75 & -71.81 & 102.50 & 299.42 & 195.04 \\
Tetrachloroethylene & $1.25 \mathrm{E}-02$ & $6.10 \mathrm{E}-03$ & $3.59 \mathrm{E}-01$ & $5.16 \mathrm{E}-08$ & $4.38 \mathrm{E}-07$ \\
Change ratio (\%) & 96.72 & -72.98 & 62.66 & 135.81 & 190.67 \\
N-bromopropane & $1.35 \mathrm{E}-02$ & $5.83 \mathrm{E}-01$ & $1.05 \mathrm{E}+00$ & $1.70 \mathrm{E}-08$ & $4.37 \mathrm{E}-07$ \\
Change ratio (\%) & 112.18 & 2484.36 & 374.82 & -22.54 & 190.04 \\
\hline
\end{tabular}

It can be seen from the results of the LCA analysis (Table 18) that although trichloroethylene has a small carbon footprint and ionizing radiation, it is not selected for use because it is a flammable liquid that can cause fire and explosion when exposed to open flames and high heat. In comparison, the carbon footprints of the commonly used single-solvent electrified cleaning agents of tetrachloroethylene and n-bromopropane are $103.8 \%$ and $178.7 \%$ higher than the carbon footprint of the electrified cleaning agent of this study. And most of the other LCA indicators of these two electrified cleaning agents are also higher. Therefore, the production of the electrified cleaning agent developed in this study has its own advantages in terms of carbon footprint and other environmental impact indicators.

\subsection{The Impact of Waste Solvent Recovery Efficiency on LCA Indicators}

In the benchmark model, the recovery ratio of waste solvents is assumed to be $80 \%$, i.e., $20 \%$ of tetrachloroethylene and D 40 solvent oil are discharged as air emissions. Although the emission of D40 solvent oil is environmentally friendly, the emission of tetrachloroethylene has an obvious impact on photochemical ozone formation potential (POFP), ecological toxicity (ET), human toxicity-carcinogenic (HT-cancer), and human body Toxicity-non-carcinogenic (HT-non cancer) indicators. Moreover, the recovery efficiency is directly related to the waste solvent recovery process. Therefore, it is necessary to further study the influence of waste solvent recovery efficiency on various indicators of the whole life cycle.

Table 19. The effect of electrified cleaning agent recovery ratio on LCA indicators

\begin{tabular}{cccccc}
\hline $\begin{array}{c}\text { Waste solution } \\
\text { recovery ratio } \\
\text { discharge ratio (\%) }\end{array}$ & GWP & AP & EP & RI & ODP \\
\hline $\mathbf{1 0 0 / 0}$ & $2.13 \mathrm{E}+00$ & $9.77 \mathrm{E}-03$ & $1.20 \mathrm{E}-03$ & $2.21 \mathrm{E}-03$ & $4.91 \mathrm{E}-05$ \\
$\mathbf{8 0 / 2 0}$ & $2.08 \mathrm{E}+00$ & $9.49 \mathrm{E}-03$ & $1.18 \mathrm{E}-03$ & $2.13 \mathrm{E}-03$ & $4.91 \mathrm{E}-05$ \\
Change ratio (\%) & -2.20 & -2.89 & -2.04 & -3.51 & 0.00 \\
$\mathbf{5 0 / 5 0}$ & $2.03 \mathrm{E}+00$ & $9.12 \mathrm{E}-03$ & $1.14 \mathrm{E}-03$ & $2.03 \mathrm{E}-03$ & $4.91 \mathrm{E}-05$ \\
Change ratio (\%) & -4.74 & -6.63 & -4.76 & -8.03 & 0.00 \\
$\mathbf{2 0 / 8 0}$ & $1.97 \mathrm{E}+00$ & $8.73 \mathrm{E}-03$ & $1.11 \mathrm{E}-03$ & $1.93 \mathrm{E}-03$ & $4.91 \mathrm{E}-05$ \\
Change ratio (\%) & -7.46 & -10.62 & -7.63 & -12.85 & 0.00 \\
$\mathbf{0} / \mathbf{1 0 0}$ & $1.90 \mathrm{E}+00$ & $8.46 \mathrm{E}-03$ & $9.87 \mathrm{E}-04$ & $1.85 \mathrm{E}-03$ & $4.91 \mathrm{E}-05$ \\
Change ratio (\%) & -10.91 & -13.45 & -17.74 & -16.29 & 0.00 \\
\hline
\end{tabular}


Table 19. The effect of electrified cleaning agent recovery ratio on LCA indicators (continued)

\begin{tabular}{cccccc}
\hline $\begin{array}{l}\text { Waste solution } \\
\text { recovery ratio } \\
\text { /discharge ratio (\%) }\end{array}$ & POFP & IRP & ET & HT-cancer & $\begin{array}{c}\text { HT-non } \\
\text { cancer }\end{array}$ \\
\hline $\mathbf{1 0 0 / 0}$ & $2.57 \mathrm{E}-02$ & $3.40 \mathrm{E}-02$ & $2.23 \mathrm{E}-01$ & $2.20 \mathrm{E}-08$ & $1.51 \mathrm{E}-07$ \\
$\mathbf{8 0 / 2 0}$ & $2.89 \mathrm{E}-02$ & $3.16 \mathrm{E}-02$ & $2.69 \mathrm{E}-01$ & $4.32 \mathrm{E}-08$ & $2.31 \mathrm{E}-07$ \\
【Change ratio】/\% & 12.25 & -7.07 & 20.97 & 96.28 & 53.08 \\
$\mathbf{5 0 / 5 0}$ & $3.36 \mathrm{E}-02$ & $2.85 \mathrm{E}-02$ & $3.40 \mathrm{E}-01$ & $7.51 \mathrm{E}-08$ & $3.51 \mathrm{E}-07$ \\
【Change ratio】/\% & 30.74 & -15.98 & 52.62 & 241.46 & 133.11 \\
$\mathbf{2 0 / 8 0}$ & $3.83 \mathrm{E}-02$ & $2.53 \mathrm{E}-02$ & $4.10 \mathrm{E}-01$ & $1.07 \mathrm{E}-07$ & $4.71 \mathrm{E}-07$ \\
【Change ratio】/\% & 49.03 & -25.57 & 83.93 & 385.15 & 212.32 \\
$\mathbf{0 / 1 0 0}$ & $4.15 \mathrm{E}-02$ & $2.30 \mathrm{E}-02$ & $4.57 \mathrm{E}-01$ & $1.28 \mathrm{E}-07$ & $5.51 \mathrm{E}-07$ \\
【Change ratio】/\% & 61.31 & -32.33 & 105.03 & 482.17 & 265.79 \\
\hline
\end{tabular}

From the data in Table 19, the recovery ratio of electrified cleaning agent is directly related to the power consumption of the waste process and the toxicity caused by environmental emissions. The higher the recovery ratio, the higher the energy consumption of the solution recovery process, which in turn leads to an increase in the carbon footprint (GWP), AP, EP, RI and IRP indicators of the whole life cycle. On the contrary, if the solvent is allowed to discharge to the environment, it will cause a significant increase in environmental toxicity indicators such as POFP, ET, HT-cancer and HT-non cancer. Therefore, the full cycle emissions of any product and process are closely related to the technical details of the entire process. The goal of reducing carbon footprint and other environmental impact indicators can be achieved only by choosing a low-emission and reasonable process.

As far as this process is concerned, a low-energy waste solvent recovery process should be developed, the power consumption of the current distillation recovery device should be reduced as much as possible, and renewable energy can be used to replace fossil energy. At the same time, the solvent recovery device needs to be improved to increase efficiency and minimize direct environmental emissions.

\subsection{Estimation of the Carbon Footprint of the Upstream Tetrachloroethylene Production Process}

The study further estimated the carbon emissions of the two upstream production processes (tetrachloroethylene and D40 solvent oil) that have the largest impact on the carbon footprint. Tetrachloroethylene (molecular formula $\mathrm{C}_{2} \mathrm{Cl}_{4}$, CAS-No. 127-18-4, also known as PCE or perchloroethylene) is a colorless and transparent liquid with an esterlike smell. It is not flammable and has no measurable flash point or flammability limit in air. Usually acetylene or ethylene is used as a raw material, and it is co-produced with trichloroethylene through chlorination and oxidation. The life cycle data of tetrachloroethylene comes from the Ecoinvent3.1 database of Europe, and the unit is 1 $\mathrm{kg}$. The boundary map is as follows: 


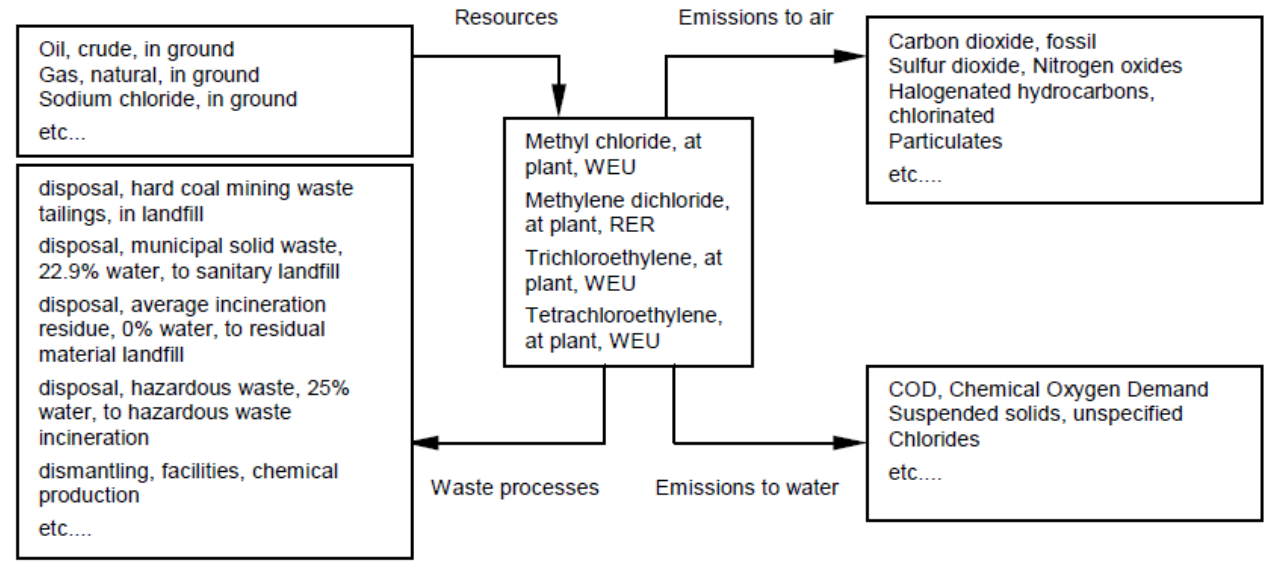

Figure 14. Process chain for the production of methyl chloride, methylene chloride, trichloroethylene and tetrachloroethylene

The chemical equation produced by tetrachloroethylene is as follows:

$$
\mathrm{C}_{2} \mathrm{H}_{4}+\mathrm{C}_{2} \mathrm{H}_{4} \mathrm{Cl}_{2}+2.5 \mathrm{Cl}_{2}+1.75 \mathrm{O}_{2} \rightarrow \mathrm{C}_{2} \mathrm{HCl}_{3}+\mathrm{C}_{2} \mathrm{Cl}_{4}+3.5 \mathrm{H}_{2} \mathrm{O}
$$

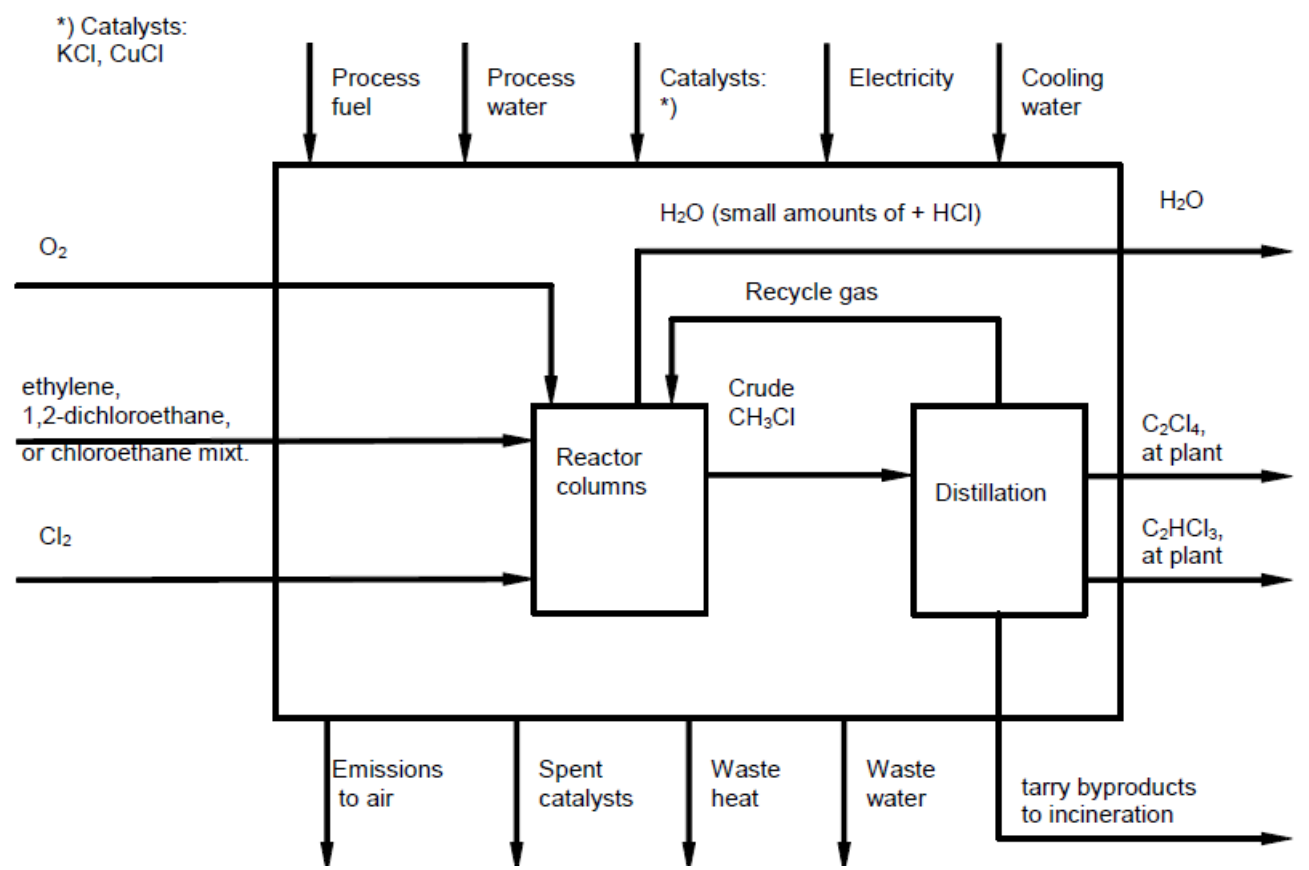

Figure 15. Simplified process for preparing tetrachloroethylene through the oxychlorination reaction (reactor) 


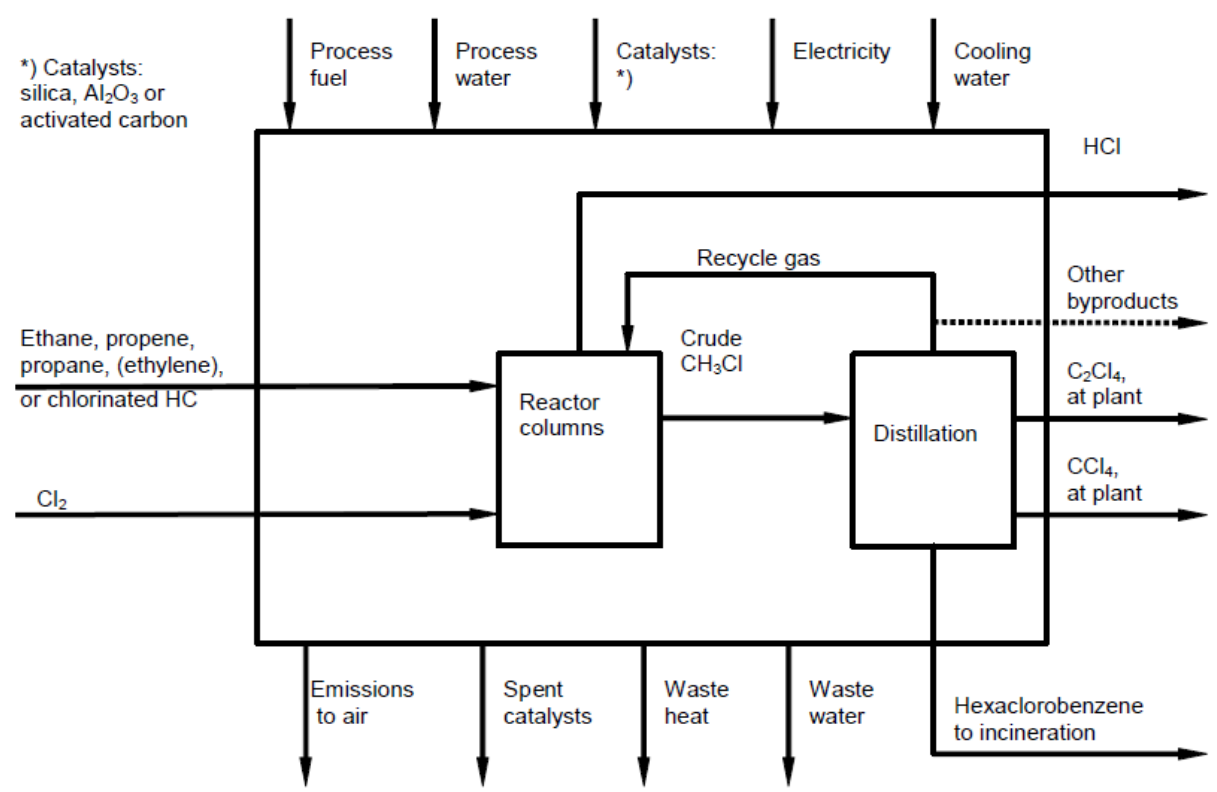

Figure 16. The process of preparing tetrachloroethylene by chlorinating solubilization

Due to the confidentiality of commercial databases, the possible sources of upstream emissions can only be inferred through possible raw materials and reaction pathways. Table 20 summarizes the carbon emission (GWP) data of $1 \mathrm{~kg}$ of ethylene $\left(\mathrm{C}_{2} \mathrm{H}_{2}\right)$, chlorine $\left(\mathrm{Cl}_{2}\right)$, and tetrachloroethylene.

Table 20. Carbon footprint analysis of tetrachloroethylene production process (unit: kg)

\begin{tabular}{|c|c|c|c|c|c|}
\hline Process & $\begin{array}{c}\text { Carbon } \\
\text { emission }\end{array}$ & Process & $\begin{array}{c}\text { Carbon } \\
\text { emission }\end{array}$ & Process & $\begin{array}{r}\text { Carbon } \\
\text { emission }\end{array}$ \\
\hline \multirow[t]{2}{*}{$\begin{array}{c}\text { Tetrachloroeth } \\
\text { ylene } \mathrm{C}_{2} \mathrm{Cl}_{4} \\
(1 \mathrm{~kg})\end{array}$} & $3.83^{b}$ & Ethylene $(1 \mathrm{~kg})$ & $1.08^{\mathrm{c}}$ & $\begin{array}{l}\text { Petroleum refined } \\
\text { products }(1 \mathrm{~kg})\end{array}$ & $0.52^{c}$ \\
\hline & & $\begin{array}{l}\text { Natural gas } \\
\text { production }\end{array}$ & 0.52 & Crude oil extraction & 0.22 \\
\hline \multirow[t]{4}{*}{ Ethylene (1 kg) } & $1.78^{\mathrm{a}}$ & $\begin{array}{l}\text { Natural gas } \\
\text { heating }\end{array}$ & 0.347 & electricity & 0.108 \\
\hline & $1.37^{\mathrm{b}}$ & $\begin{array}{l}\text { Petroleum } \\
\text { Refined } \\
\text { Products }\end{array}$ & 0.14 & By sea & 0.0896 \\
\hline & $1.08^{\mathrm{c}}$ & & & $\begin{array}{l}\text { Residual fuel oil } \\
\text { combustion }\end{array}$ & 0.0843 \\
\hline & & & & Natural gas heating & 0.022 \\
\hline Chlorine (1 kg) & $1.68-2.51^{\mathrm{a}}$ & Chlorine $(1 \mathrm{~kg})$ & $1.04^{\mathrm{c}, \mathrm{d}}$ & & \\
\hline process & $1.32-1.34^{\mathrm{b}}$ & electricity & 0.569 & & \\
\hline \multirow{2}{*}{$\begin{array}{c}\text { Tetrachloroeth } \\
\text { ylene } \mathrm{C}_{2} \mathrm{Cl}_{4} \\
(1 \mathrm{~kg})\end{array}$} & $1.04^{\mathrm{c}, \mathrm{d}}$ & natural gas & 0.257 & & \\
\hline & & $\mathrm{NaCl}$ & 0.136 & & \\
\hline Ethylene (1 kg) & & Coal & 0.07 & & \\
\hline
\end{tabular}

Data source a: CLCD-China-ECER 0.8; b: Ecoinvent 3.1; c: USLCI; d: commercial database 
Emissions from ethylene production originate from natural gas production, process heating, and oil refining. However, according to the analysis of the molecular formula and chemical reaction formula of tetrachloroethylene, ethylene production does not contribute much to the carbon footprint of the entire process. The main carbon emission of tetrachloroethylene comes from the chlorine production process. According to the production methods of different countries and regions, it can be seen that China uses the ion-exchange membrane method of chlor-alkali electrolysis to produce chlorine, producing carbon emissions of 1.68-2.51 $\mathrm{kg} \mathrm{CO}_{2}$ eq. The chlor-alkali electrolysis process (membrane technology), which is widely used in Europe and the United States, has a lower carbon emission of $1.04 \mathrm{~kg} \mathrm{CO}_{2}$ eq. According to the LCA boundary map (Figure 17) and analysis results given by the commercial database, it can be seen that the carbon footprint sources of the production of chlorine include electricity, natural gas combustion and the energy consumption of the $\mathrm{NaCl}$ production process.

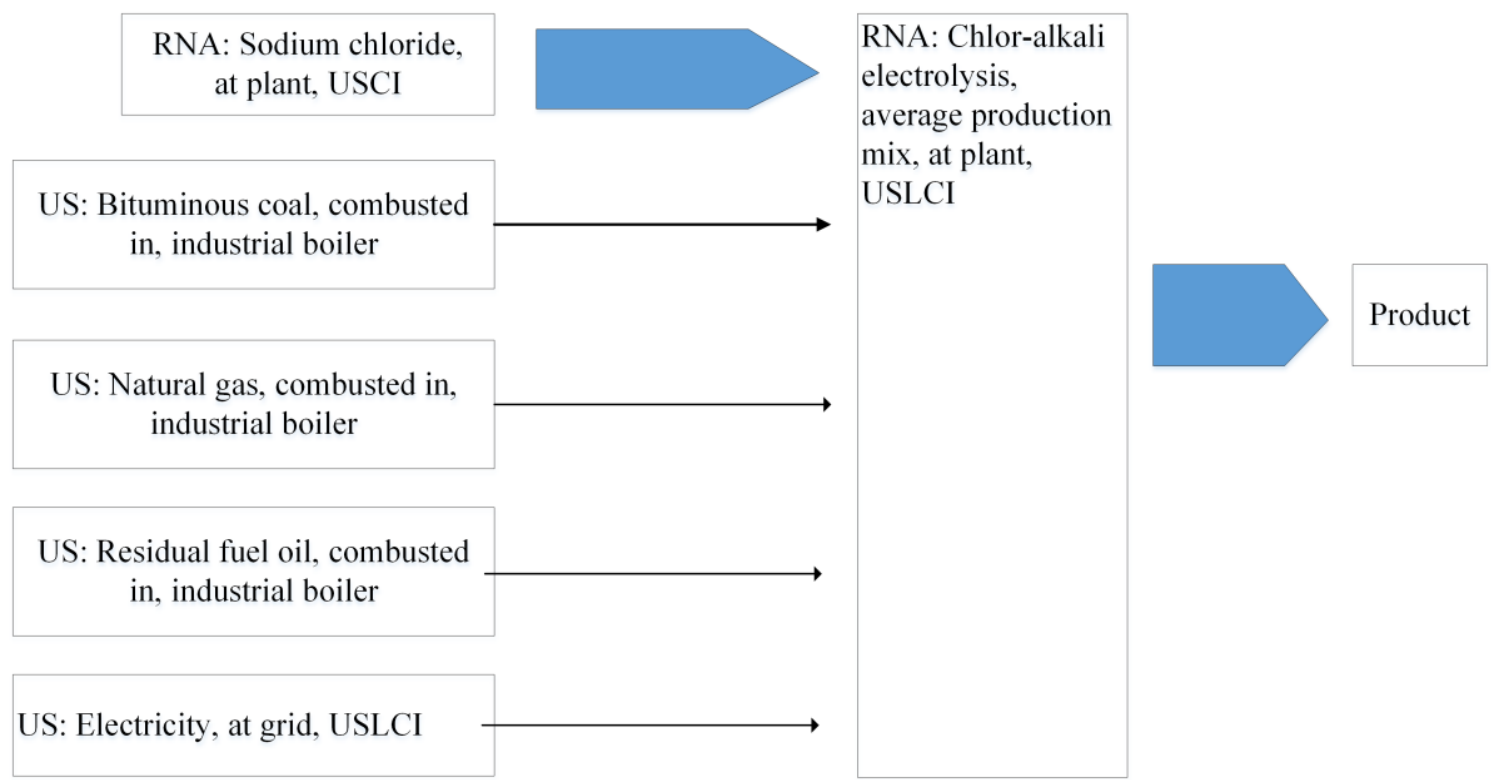

Figure 17. LCA boundary diagram of chlorine produced by electrolysis

The analysis shows that the main carbon emissions from the tetrachloroethylene production process are the electricity consumption of each process, the combustion of natural gas production and the combustion of other energy materials for heating. It is recommended to use renewable raw materials instead of crude oil to obtain carbon credits based on geographical advantages, and try to use production processes with lower carbon emissions, while the exhaust gas from the traditional production process is strictly absorbed and purified before being discharged.

\subsection{Carbon Footprint Estimation of the Upstream D40 Solvent Oil Production Process}

D40 solvent oil is made of distillate oil as raw material, after high-pressure hydrorefining and fractional distillation. Its main component is saturated alkanes, including nonane, decane and undecane. The production process is basically the same as naphtha. So in this study, the naphtha process was used instead of D40 solvent oil. The carbon footprint of the naphtha production process is estimated using data from 
commercial databases. The process diagram is shown in Figure 18, and the data of several databases is summarized in Table 21 .

It can be seen from the process diagram that petroleum refining is a complex process. The data set includes drilling, crude oil production, processing, transportation of crude oil through pipelines, and ship transportation to the refinery. The whole process requires a lot of fuel to generate heat, steam and electricity. These fuels may be refinery gas, heating oil (residual oil), gasoline coke, middle distillate oil and liquefied petroleum gas. The output of petroleum refining is a series of products, and the quality of the product is closely related to the composition of the crude oil, the region and the refining technology used.

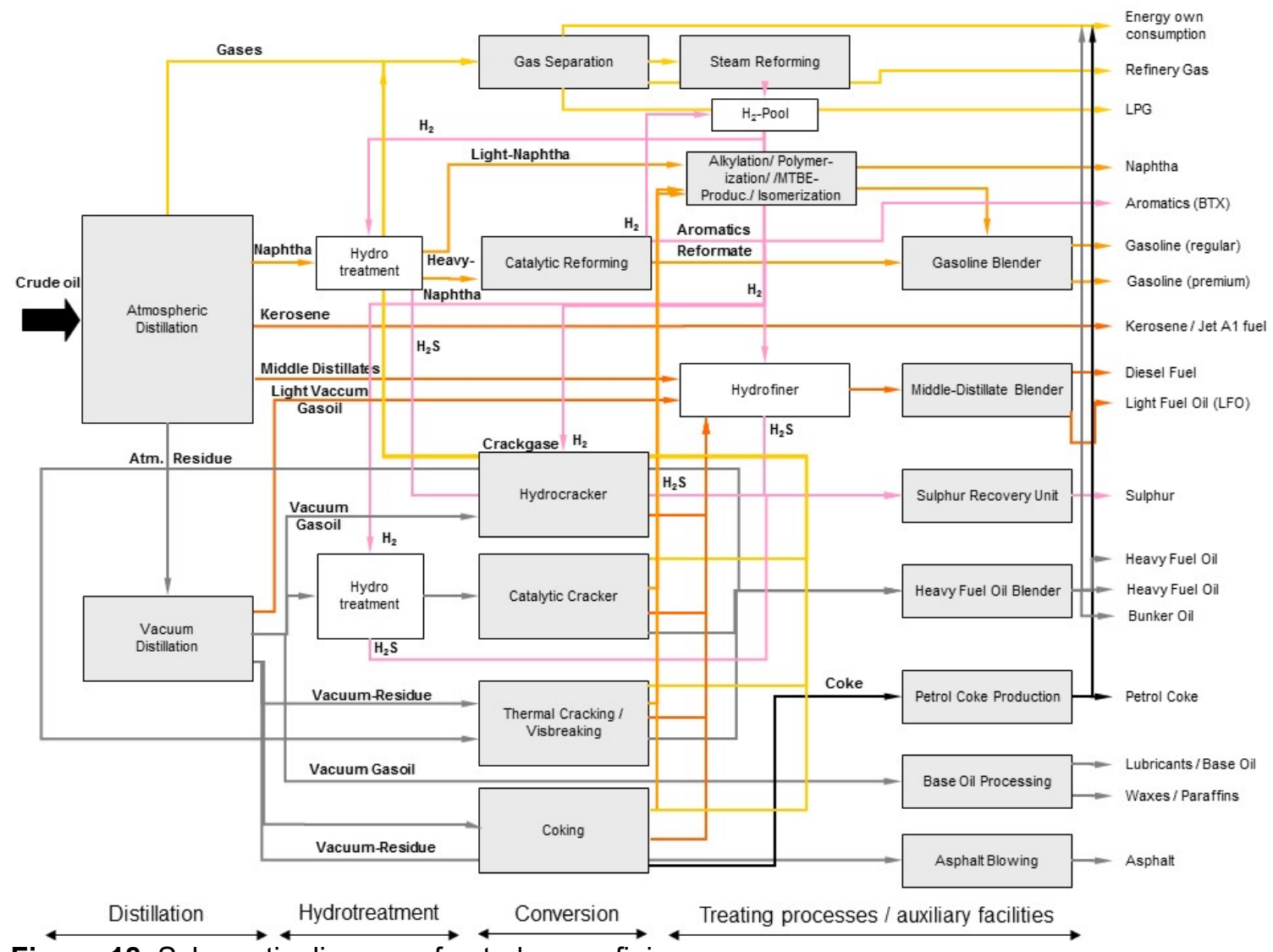

Figure 18. Schematic diagram of petroleum refining process

Table 24. Carbon footprint analysis of D40 solvent oil (naphtha) production process (Unit: kg)

\begin{tabular}{cc}
\hline Process & Carbon emission \\
\hline & $0.679^{\mathbf{a}}$ \\
Naphtha (1 kg) & $0.363^{\mathbf{b}}$ \\
& $0.599^{\mathbf{c}}$
\end{tabular}

Data source: a: CLCD-China-ECER 0.8; b: Ecoinvent 3.1; c: commercial database

Analysis shows that the main carbon emissions from the production process of D40 solvent oil (naphtha) come from the production process of petroleum refining and natural gas production, and the emissions are closely related to the quality of crude oil and the refining process. It is recommended to improve the existing petroleum refining 
process, purchase cleaner crude oil as the raw material, and discharge the exhaust gas after strict absorption and purification.

\section{Life Cycle Explanation}

\subsection{Assumptions and Limitations}

This study calculated the results of 10 indicators of the electrified cleaning agent production process. The detergent formula is determined by this research and does not represent the industry average. When simulating the calculation process, only the energy consumption of the core equipment is calculated. It is assumed that when the main equipment is increased, only electricity is consumed, and the process emissions are not increased. It is assumed that the production technology used for the raw materials is the same as the technology in commercial databases and public documents, and the emissions are the same.

\subsection{Integrity Description}

Strict mass balance and energy balance calculations were carried out for the production process of the electrified cleaning agent. The emission data were adopted from the literature and company measurement. The possible increase in power consumption in the process of production improvement has been discussed in detail in the carbon footprint analysis of power consumption in the section 4.5. The emission data of the production process and waste disposal was obtained from the public databases, and the emission data is highly reliable.

\subsection{Data Quality Assessment}

The report uses the CLCD quality assessment method to complete the uncertainty assessment of the model inventory data on the eFootprint system. The data quality evaluation results obtained are shown in Table 25.

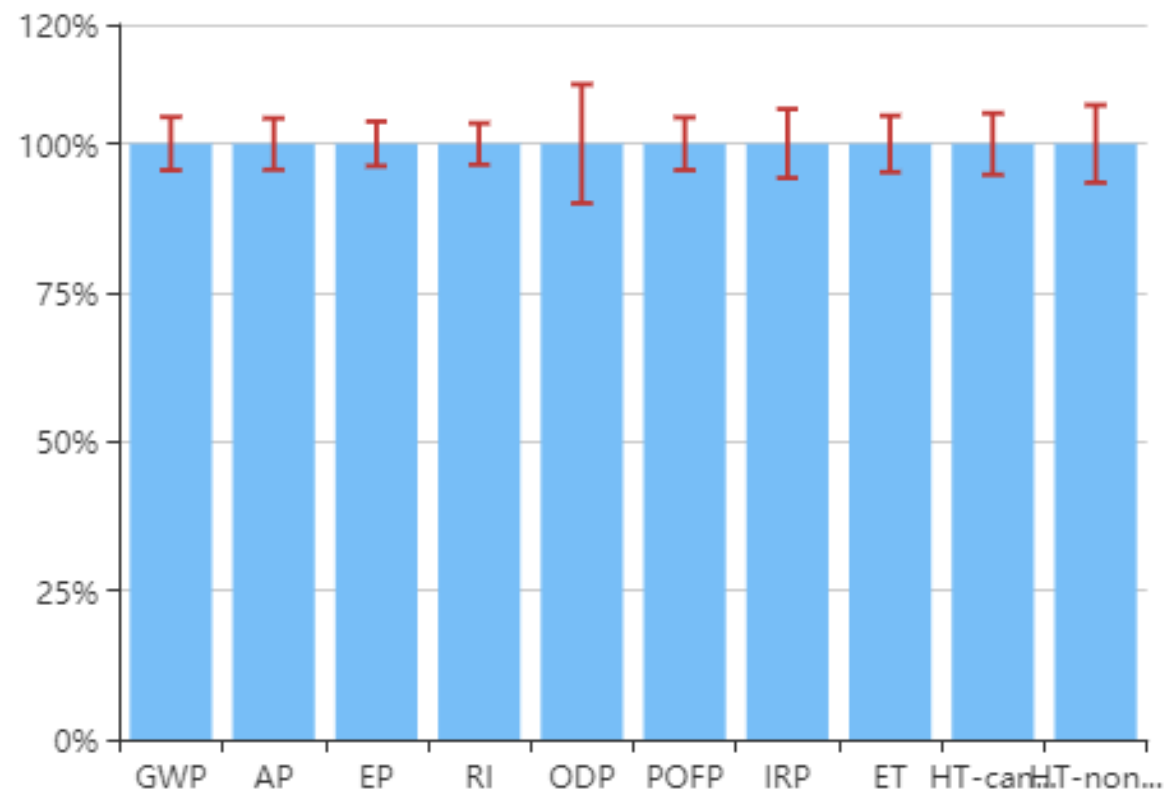

Figure 19. Uncertainty of LCA results 
Table 25. LCA data quality assessment

\begin{tabular}{|c|c|c|c|c|}
\hline Indicator name & $\begin{array}{c}\text { Abbreviation } \\
\text { (unit) }\end{array}$ & LCA results & $\begin{array}{l}\text { Uncertainty of } \\
\text { result }\end{array}$ & $\begin{array}{c}\text { Results upper and } \\
\text { lower limits } \\
\text { (95\% confidence } \\
\text { interval) } \\
\end{array}$ \\
\hline Climate change & $\begin{array}{c}\text { GWP }\left(\mathrm{kg} \mathrm{CO} \mathrm{CO}_{2}\right. \\
\text { eq) }\end{array}$ & $2.084 \mathrm{E}+000$ & $4.44 \%$ & {$[1.99,2.18]$} \\
\hline Acidification & $\begin{array}{c}\mathrm{AP}(\mathrm{kg} \mathrm{SO} \\
\mathrm{eq})\end{array}$ & $9.494 \mathrm{E}-003$ & $4.31 \%$ & {$[9.08 \mathrm{E}-03,9.90 \mathrm{E}-03]$} \\
\hline $\begin{array}{c}\text { Eutrophication } \\
\text { Potential }\end{array}$ & $\begin{array}{c}\mathrm{EP}\left(\mathrm{kg} \mathrm{PO}_{4}{ }^{3-}\right. \\
\mathrm{eq})\end{array}$ & $1.175 \mathrm{E}-003$ & $3.75 \%$ & {$[1.13 \mathrm{E}-03,1.22 \mathrm{E}-03]$} \\
\hline $\begin{array}{c}\text { Inhalable } \\
\text { inorganic matter }\end{array}$ & $\begin{array}{l}\mathrm{RI}(\mathrm{kg} \text { PM } 2.5 \\
\text { eq) }\end{array}$ & $2.136 \mathrm{E}-003$ & $3.46 \%$ & {$[2.06 \mathrm{E}-03,2.21 \mathrm{E}-03]$} \\
\hline Ozone depletion & $\begin{array}{c}\text { ODP }(\mathrm{kg} \\
\text { CFC-11 eq) }\end{array}$ & 4.909E-005 & $9.95 \%$ & [4.42E-05,5.39E-05] \\
\hline $\begin{array}{l}\text { Photochemical } \\
\text { ozone synthesis }\end{array}$ & $\begin{array}{c}\text { POFP (kg } \\
\text { NMVOC eq) }\end{array}$ & $2.886 \mathrm{E}-002$ & $4.39 \%$ & {$[0.03,0.03]$} \\
\hline $\begin{array}{l}\text { Ionizing } \\
\text { radiation }\end{array}$ & $\begin{array}{l}\text { IRP (kg U235 } \\
\text { eq) }\end{array}$ & $3.161 \mathrm{E}-002$ & $5.79 \%$ & {$[0.03,0.03]$} \\
\hline Ecotoxicity & ET (CTUe) & 2.694E-001 & $4.73 \%$ & {$[0.26,0.28]$} \\
\hline $\begin{array}{l}\text { Human toxicity- } \\
\text { carcinogenic }\end{array}$ & $\begin{array}{l}\text { HT-cancer } \\
\text { (CTUh) }\end{array}$ & $4.316 \mathrm{E}-008$ & $5.15 \%$ & {$[4.09 \mathrm{E}-08,4.53 \mathrm{E}-08]$} \\
\hline $\begin{array}{l}\text { Human toxicity- } \\
\text { non-carcinogen }\end{array}$ & $\begin{array}{c}\text { HT-non } \\
\text { cancer } \\
\text { (CTUh) }\end{array}$ & 2.307E-007 & $6.49 \%$ & {$[2.15 \mathrm{E}-07,2.45 \mathrm{E}-07]$} \\
\hline
\end{tabular}

\section{Conclusions}

This study analyzed the whole life cycle of the electrified cleaning agent developed. This electrified cleaning agent has the characteristics of non-phosphorus, green and environmental protection, and very broad market prospects. The life cycle model is cradle to grave, and the background data sets include power grid, transportation, high-density polyethylene, chemicals, etc. The production process uses computer simulation to carry out strict mass balance and energy consumption calculations. The study integrated the data from the manufacturer and the contents of the public literature to analyze the emissions.

(1) The analysis of the whole life cycle model of the established model shows that the GWP of the life cycle of $1 \mathrm{~kg}$ of electrified cleaning agent is $2.08 \mathrm{~kg} \mathrm{CO}_{2} \mathrm{eq}$, AP is $9.49 \mathrm{E}-03 \mathrm{~kg} \mathrm{SO}{ }_{2}$ eq, EP is $1.18 \mathrm{E}-03 \mathrm{~kg} \mathrm{PO}_{4}{ }^{3-}$ eq, and RI is $2.13 \mathrm{E}-03 \mathrm{~kg} \mathrm{PM} 2.5 \mathrm{eq}$, ODP is $4.91 \mathrm{E}-05 \mathrm{~kg}$ CFC-11 eq, POFP is $2.89 \mathrm{E}-02 \mathrm{~kg}$ NMVOC eq, IRP is $3.16 \mathrm{E}-02$ $\mathrm{kg} \mathrm{U} 235 \mathrm{eq}$, ET is 2.69E-01 CTUe, HT-cancer is 4.32E-08 CTUh, and HT-non cancer is $2.31 \mathrm{E}-07 \mathrm{CTUh}$. The uncertainty of the results is between $3.46-9.95 \%$.

(2) The four processes of tetrachloroethylene production, D40 solvent oil production, tetrachloroethylene environmental discharge 【 use 】 and electricity 【 product disposal】 have significant effects on each LCA indicator, so they are the focus of process improvement.

(3) Changes in power consumption during production and transportation distance of raw materials have little effect on total carbon emissions. 
(4) Compared with the production process of single-solvent electrified cleaning agent tetrachloroethylene and n-bromopropane, the production of the electrified cleaning agent developed in this study has its own advantages in terms of carbon footprint and other environmental impact indicators.

(5) The higher the waste solvent recovery ratio, the higher the energy consumption of the solution recovery process, which in turn leads to an increase in the carbon footprint (GWP), AP, EP, RI and IRP indicators of the whole life cycle. On the contrary, if the solvent is allowed to discharge to the environment, it will cause a significant increase in environmental toxicity indicators such as POFP, ET, HTcancer and HT-non cancer. It is necessary to develop a low-energy waste solvent recovery process, or improve the current distillation equipment to reduce energy consumption. At the same time, the solvent recovery device needs to be improved to increase efficiency and minimize direct environmental emissions.

(6) Estimates of carbon emissions from major upstream production processes show that carbon emissions mainly come from the power consumption of each process, natural gas production and combustion, and other energy materials for heating. It is recommended to use renewable raw materials instead of crude oil to obtain carbon credits based on geographical advantages, and try to use production processes with lower carbon emissions, while the exhaust gas from the traditional production process is strictly absorbed and purified before being discharged.

\section{Suggestions}

(1) The results of this study are not industry average, and the improved formula needs to be recalculated.

(2) It is recommended to improve the existing petroleum refining process; purchase cleaner crude oil as raw materials; use renewable raw materials instead of crude oil to obtain carbon credits according to geographical advantages; and exhaust gas from the production process after strict absorption and purification before being discharged.

(3) It is also necessary to pay attention to the recycling of packaging materials.

\section{ACKNOWLEDGMENTS}

We are grateful for the support from the School of Chemical Engineering and Pharmacy at the Wuhan Institute of Technology. We would also like to thank Mr. Rui Li from Mississippi State University for his assistance in the simulation of chemical processes.

\section{CONFLICTS OF INTEREST}

The authors declare that there is no conflict of interests regarding the publication of this paper. 


\section{REFERENCES}

[1] Rong, X. (2004) Key Performances and Developing Ideas of Cleanser for Electrified Cleaning. Cleaning Technology.

[2] Ecoinvent 3.1. (other countries). Alex Primas.2014-11-13.

[3] CLCD-China-ECER 0.8. Naphtha/Naphtha-Naphtha/Naphtha-Atmospheric Vacuum (China). He Qin.2013-02-09

[4] CLCD-China-ECER 0.8. Isopropanol-(China). Fan Cidong. 2013-02-09

[5] CLCD-China-ECER 0.8. East China Power Grid-East China Power Transmission (China). HouPing.2013-02-09.

[6] CLCD-China-ECER 0.8. Truck transportation-heavy diesel truck transportation (30t)-China (China).yangjie.2013-02-09

Article copyright: ( 2021 Peng Liu, Bo Zhang, Changyan Yang, Yu Gong, Yinhang Qu, Jiayi Li, Bohan Yang, and Yigang Ding. This is an open access article distributed under the terms of the Creative Commons Attribution 4.0 International License, which permits unrestricted use and distribution provided the original author and source are credited. 